\title{
Freudenthal Gauge Theory
}

\section{Alessio Marrani, ${ }^{a}$ Cong-Xin Qiu, ${ }^{b}$ Sheng-Yu Darren Shih, ${ }^{c}$ Anthony Tagliaferro ${ }^{c}$ and Bruno Zumino ${ }^{c, d}$}

${ }^{a}$ Theory division, CERN, CH 1211, Geneva 23, Switzerland

${ }^{b}$ School of Physics and Astronomy, University of Minnesota/Twin Cities, Minneapolis, MN 55455, U.S.A.

${ }^{c}$ Department of Physics and Center for Theoretical Sciences, University of California, Berkeley, CA 94720-7300, U.S.A.

${ }^{d}$ Lawrence Berkeley National Laboratory, Theory Group, Berkeley, CA 94720-8162, U.S.A.

E-mail: alessio.marrani@cern.ch, congxin.qiu@gmail.com, atag@berkeley.edu, s.y.darren.shih@berkeley.edu, zumino@thsrv.lbl.gov

AbSTRACT: We present a novel gauge field theory, based on the Freudenthal Triple System $(F T S)$, a ternary algebra with mixed symmetry (not completely symmetric) structure constants. The theory, named Freudenthal Gauge Theory $(F G T)$, is invariant under two (off-shell) symmetries: the gauge Lie algebra constructed from the FTS triple product and a novel global non-polynomial symmetry, the so-called Freudenthal duality.

Interestingly, a broad class of FGT gauge algebras is provided by the Lie algebras "of type $\mathfrak{e}_{7}$ " which occur as conformal symmetries of Euclidean Jordan algebras of rank 3 , and as $U$-duality algebras of the corresponding (super)gravity theories in $D=4$.

We prove a No-Go Theorem, stating the incompatibility of the invariance under Freudenthal duality and the coupling to space-time vector and/or spinor fields, thus forbidding non-trivial supersymmetric extensions of FGT.

We also briefly discuss the relation between FTS and the triple systems occurring in BLG-type theories, in particular focusing on superconformal Chern-Simons-matter gauge theories in $D=3$.

Keywords: Gauge Symmetry, Global Symmetries, Chern-Simons Theories

ARXIV EPRINT: 1208.0013 


\section{Contents}

1 Introduction 1

2 Freudenthal Triple Systems (FTS's) 4

2.1 Rank-3 Jordan algebras and Lie algebras 4

2.2 General case 5

2.3 Axiomatic definition 6

$\begin{array}{lll}2.4 & \text { FTS } \text { structure constants and their invariance } & 7\end{array}$

2.5 Freudenthal duality 8

3 Freudenthal Gauge Theory (FGT) 11

$\begin{array}{lll}3.1 & \text { From global symmetry... } & 11\end{array}$

$\begin{array}{lll}3.2 & \text {...to gauge symmetry } & 12\end{array}$

$\begin{array}{ll}3.3 \text { The Lagrangian } & 14\end{array}$

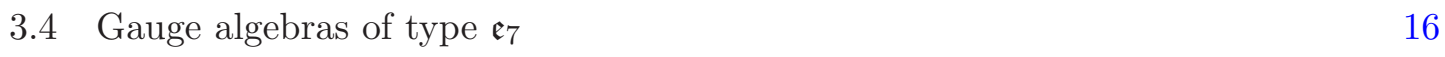

$\begin{array}{lll}3.5 & \text { FGT and supergravity } & 17\end{array}$

4 Generalization? $\quad 20$

$\begin{array}{ll}4.1 \text { Coupling to a vector space } & 20\end{array}$

$\begin{array}{ll}4.2 \text { A no-go theorem } & 22\end{array}$

$5 \quad$ FGT and $(\mathcal{N}=3, D=3)$ SC CSM gauge theories 23

$\begin{array}{lll}6 & \text { Concluding remarks } & 28\end{array}$

$\begin{array}{ll}\text { A Freudenthal duality } & 30\end{array}$

B Space-time symmetry of scalar kinetic term 31

$\begin{array}{lll}\text { C Axioms of } \mathfrak{V} & 32\end{array}$

\section{Introduction}

The idea that a ternary algebra might be an essential structure of physical theories has a long history.

In the early 70's, Nambu [1] proposed a generalized Hamiltonian system based on a ternary product, the Nambu-Poisson bracket. Despite some partial results (see e.g. [2] for a comprehensive review), the quantization of the Nambu-Poisson bracket remains a long-term puzzle.

However, ternary algebras and their applications to theoretical physics have been object of intense study over the last four decades. The Jordan triple product was exploited by Günaydin and Gürsey in their quest for a formulation of quantum mechanics over different division algebras, including octonions; this investigation led to the quadratic Jordan 
formulation of quantum mechanics in terms of Jordan triple product $[3,4]$ that extends to the octonionic quantum mechanics of [5], which has no formulation over an Hilbert space. Later on, a unified construction of Lie algebras and Lie superalgebras over triple systems was achieved by Bars and Günaydin in [6], and in [7] various composite models based on ternary algebras were investigated. Two-dimensional superconformal algebras over triple systems were then constructed in [8]; in particular, Freudenthal triple systems were applied to $\mathcal{N}=4$ superconformal algebras and gauged WZW models in [9].

Recently, ternary algebras re-appeared in the study of $M$-theory by Bagger and Lambert [10] and by Gustavsson [11], in which a ternary Lie-3 algebra is proposed as the underlying gauge symmetry structure on a stack of supersymmetric M2-branes; this is the famous BLG theory (for a recent review and list of refs., see e.g. [12]). When taking the Nambu-Poisson bracket as an infinite-dimensional generalization of the Lie-3 bracket, one gets from the BLG theory a novel six-dimensional field theory, which can be interpreted as a non-commutative version of the M5-brane theory [13].

In the present paper, we propose a novel gauge field theory, based on another ternary algebra: the Freudenthal Triple System ${ }^{1}$ (FTS). We call this theory "Freudenthal Gauge Theory" (FGT). In its simplest setup, FGT contains a bosonic scalar field $\phi(x)$ valued in the FTS $\mathfrak{K}$ together with a gauge field $A_{\mu}(x)$ taking values in the symmetric product $\mathfrak{K} \otimes_{s} \mathfrak{K}$. Similar to the BLG theory, the gauge transformation is constructed from a triple product defined over the FTS $\mathfrak{K}$. However, unlike the totally anti-symmetric Lie-3 bracket used in the BLG theory, in general the FTS triple product does not have a simple symmetry structure with respect to the exchange of a pair of its arguments. Nevertheless, one can still prove that the gauge invariance of FGT is guaranteed by the algebraic properties of the FTS.

Besides the off-shell gauge symmetry, FGT also possesses a novel global (off-shell) symmetry, the so-called Freudenthal duality (F-duality). This is a non-linear, non-polynomial mapping from $\mathfrak{K}$ to $\mathfrak{K}$, relying on non-linear identities which can be traced back to the early days of the mathematical investigation of FTS's [22]. The name Freudenthal duality is much more recent, and it was introduced within physical literature in [23], in the study of Maxwell-Einstein supergravity theories (MESGT's) in $D=4$ space-time dimensions based on symmetric scalar manifolds and with non-degenerate groups of type $E_{7}[22-28]$ as generalized electric-magnetic $\left(U\right.$-)duality ${ }^{2}$ symmetries. In such a framework, F-duality was observed as a non-polynomial, anti-involutive mapping on $\mathfrak{K}$-valued black hole charges (i.e.

\footnotetext{
${ }^{1}$ Historically, there are several different notions of Freudenthal Triple System, which differ by the symmetry structure of their triple product. They were introduced in mathematics in order to address different algebraic properties of the triple system. Although simply related, different definitions of FTS have different properties, which of course can be translated from one to another. In the physics literature, the FTS we focus on in this paper is sometimes also called generalized Freudenthal Triple System, which makes the derivation property more transparent.

Since there is no general agreement on the definition, we will simple denote the triple system in this paper by Freudenthal Triple System (FTS). The FTS introduced in $\mathcal{N}=2$ Maxwell-Einstein supergravity and its $\mathcal{N}>2$ generalizations [14, 15] (see also e.g. [19] and [20, 21] for recent reviews) can be regarded as some special cases of it.

${ }^{2}$ Here $U$-duality is referred to as the "continuous" symmetries of $[29,30]$. Their discrete versions are the $U$-duality non-perturbative string theory symmetries introduced in [31].
} 
fluxes of the Abelian 2-form field strengths) which keeps the Bekenstein-Hawking [32, 33] black hole entropy invariant [23]. Further generalization to a generic $\mathcal{N}=2$ special Kähler geometry, to its $\mathcal{N}>2$ generalization and to the so-called effective black hole potential governing the scalar flows has been discussed in [24].

At any rate, FGT, in its simplest setup presented in this paper, can be regarded as the simplest gauge theory admitting F-duality as global symmetry. Despite the No-Go theorem proved in section 4.2, a slight generalization of the FGT will be presented in a companion paper [34].

Intriguingly, as discussed in section 5, FGT shares the same symmetry structures as the "quaternionic level" of Faulkner's construction [35], which relates triple systems to pairs $(\mathfrak{g}, \mathbf{V})$ of a metric Lie algebra $\mathfrak{g}$ and a suitable representation $\mathbf{V}$. After the treatment $[36,37]$, an interesting similarity between FGT and the bosonic sector of $\mathcal{N}=3$, $D=3$ superconformal (SC) Chern-Simons-matter (CSM) gauge theories can be envisaged. An important difference relies in supersymmetry, which in FGT, as discussed in section 4, is essentially spoiled by the enforcement of global invariance under F-duality; this affects also other terms in the Lagrangian, e.g. the scalar potential (quartic in FGT, sextic in BLG-type theories).

All in all, we can observe that, with some important differences pointed out along the present investigation, the same symmetry structures are shared (with different implementations and physical meanings) by three (a priori very different) classes of theories, namely: $(D=3)$ FGT (non-supersymmetric), $D=4$ MESGT (with various amounts of local supersymmetry) and $D=3$ SC CSM gauge theory (with $\mathcal{N}=3$ global supersymmetry). Further details and results will be reported in a companion paper [34].

This paper is organized as follows.

We start by recalling the relation between FTS, rank-3 Euclidean Jordan algebras and exceptional Lie algebras (section 2.1); the treatment is then generalized in section 2.2. The axiomatic definition of a FTS and the general symmetry of its structure constants are then discussed in sections 2.3 and 2.4. The Freudenthal duality for a generic FTS is introduced in section 2.5, along with a discussion of its basic properties.

The global transformation constructed from the FTS triple product is introduced in section 3.1, and its gauging is discussed in section 3.2. Then, in section 3.3 we propose a bosonic Lagrangian density that exhibits both FTS gauge transformations and (global) Fduality as off-shell symmetries, and we provide a detailed proof of its invariance under such symmetries. The class of FGT gauge Lie algebras of type $\mathfrak{e}_{7}$ is considered in section 3.4, and the intriguing relation between the corresponding FGT and $D=4$ MESGT's with $U$-duality symmetry given by such Lie algebras of type $\mathfrak{e}_{7}$ is discussed in section 3.5.

The possible generalization of the simplest FGT Lagrangian introduced in section 3.3 is discussed in section 4, in which the FTS $\mathfrak{K}$ is coupled to the most general algebraic system, and the mathematical structure required for a consistent definition of F-duality is investigated (section 4.1); a No-Go theorem is proved in section 4.2.

The intriguing similarities (and important differences) between FGT and (the bosonic sector of) $\mathcal{N}=3 \mathrm{SC}$ CSM gauge theories in $D=3$ are discussed in section 5 . 
The concluding section 6 contains a summary, along with some remarks and an outlook of further developments.

Three appendices conclude the paper. Appendices A and B respectively contain details on the F-duality and on the FGT scalar kinetic term, whereas appendix C lists the induced axioms needed for the discussion of the generalization of FGT and in the proof of the No-Go theorem of section 4.2 .

As mentioned above, further results and more detailed analysis of some topics mentioned along the paper will be reported in a companion work [34].

\section{Freudenthal Triple Systems (FTS's)}

\subsection{Rank-3 Jordan algebras and Lie algebras}

The Freudenthal Triple System (FTS) $\mathfrak{K}$ was first introduced by Freudenthal in his study of exceptional Lie algebras [38-40] (see also [41]). In the original construction, $\mathfrak{K}$ is defined to be the direct sum of two copies of a Jordan Triple System (JTS) $\mathfrak{J}$ and two copies of real numbers ${ }^{3} \mathbb{R}$ :

$$
\mathfrak{K}(\mathfrak{J}) \equiv \mathfrak{J} \oplus \mathfrak{J} \oplus \mathbb{R} \oplus \mathbb{R} .
$$

Over the vector space $\mathfrak{K}(\mathfrak{J})$, one can introduce a symplectic invariant 2 -form, as well as a triple product. The latter is defined via the completely symmetric tri-linear form (also known as cubic norm) of the JTS $\mathfrak{J}$, and it can be re-interpreted as a linear map $\mathcal{L}_{\phi_{I} \phi_{J}}$ over $\mathfrak{K}$ parametrized by a pair of elements $\phi_{I}, \phi_{J} \in \mathfrak{K}$ (cfr. definition (2.13)).

In Freudenthal's construction of exceptional Lie algebras, the JTS $\mathfrak{J}$ is restricted to a rank-3 simple Euclidean Jordan algebra $\widehat{\mathfrak{J}}$, namely $\widehat{\mathfrak{J}}=\mathbb{R}$ or $\widehat{\mathfrak{J}}=J_{3}^{\mathfrak{A}} \equiv H_{3}(\mathfrak{A})$, where $H_{3}(\mathfrak{A})$ stands for the algebra of Hermitian $3 \times 3$ matrices with entries taking values in one of the four normed division algebras $\mathfrak{A}=\mathbb{R}$ (real numbers), $\mathbb{C}$ (complex numbers), $\mathbb{H}$ (quaternions), $\mathbb{O}$ (octonions) (see e.g. [42]). Then, by introducing in $\mathfrak{K}(\widehat{\mathfrak{J}})$ the submanifold

$$
\mathfrak{M}_{\widehat{\mathfrak{J}}} \equiv\left\{\phi_{I} \in \mathfrak{K}(\widehat{\mathfrak{J}}) \mid \mathcal{L}_{\phi_{I} \phi_{I}} \phi_{J}=0, \forall \phi_{J} \in \mathfrak{K}(\widehat{\mathfrak{J}})\right\},
$$

the five exceptional (finite-dimensional) Lie algebras $\mathfrak{G}=\mathfrak{g}_{2}, \mathfrak{f}_{4}, \mathfrak{e}_{6}, \mathfrak{e}_{7}, \mathfrak{e}_{8}$ arise as the the direct sum of the algebra $\operatorname{Inv}\left(\mathfrak{M}_{\widehat{\mathfrak{J}}}\right)$ that keeps $\mathfrak{M}_{\widehat{\mathfrak{J}}}$ invariant, together with a copy of $\mathfrak{s u}(2)$ and two copies (namely, an $\mathfrak{s u}(2)$-doublet) of $\mathfrak{K}(\widehat{\mathfrak{J}})[38,43]$ :

$$
\mathfrak{G}=\operatorname{Inv}\left(\mathfrak{M}_{\widehat{\mathfrak{J}}}\right) \oplus \mathfrak{s u}(2) \oplus \mathfrak{K}(\widehat{\mathfrak{J}}) \oplus \mathfrak{K}(\widehat{\mathfrak{J}}) .
$$

As a vector space, $\mathfrak{K}(\widehat{\mathfrak{J}})$ may be regarded as the representation space of a non-trivial ${ }^{4}$ symplectic representation $\mathbf{R}$ of the algebra $\operatorname{Inv}\left(\mathfrak{M}_{\widehat{\mathfrak{J}}}\right)$ itself, introduced in (2.3):

$$
\mathfrak{K}(\widehat{\mathfrak{J}}) \sim \mathbf{R}\left(\operatorname{Inv}\left(\mathfrak{M}_{\widehat{\mathfrak{J}}}\right)\right) .
$$

\footnotetext{
${ }^{3}$ Namely, the ground field was chosen to be $\mathbb{R}$. Other choices are of course possible (such as $\mathbb{Z}$ or $\mathbb{C}$ ), but we will not deal with them in the present investigation.

${ }^{4}$ Such a representation is not necessarily the smallest one. A counter-example is provided e.g. by $\mathfrak{s p}(6)=\operatorname{Inv}\left(\mathfrak{M}_{J_{3}^{\mathbb{R}}}\right)$, whose smallest non-trivial symplectic irrep. is the fundamental 6. However, $\mathfrak{K}\left(J_{3}^{\mathbb{R}}\right)$ has dimension 14, and it is based on the rank-3 completely antisymmetric irrep. 14', which exhibits a completely symmetric rank-4 invariant structure.

However, a suitable FTS $\mathfrak{K}$ on the $\mathbf{6}$ can also be constructed; see point 2 in section 5 .
} 
At least for $\mathbf{R}$ irreducible, $\operatorname{Inv}\left(\mathfrak{M}_{\widehat{\mathfrak{J}}}\right)$ is maximally (and non-symmetrically) embedded into the symplectic algebra $\mathfrak{s p}(\mathfrak{K}(\widehat{\mathfrak{J}}))$ through the Gaillard-Zumino (GZ) embedding [44] (see also e.g. [78] for a recent review)

$$
\begin{gathered}
\mathfrak{s p}(\mathfrak{K}(\widehat{\mathfrak{J}})) \\
\operatorname{Fund}(\mathfrak{s p}(\mathfrak{K}(\widehat{\mathfrak{J}})))=\mathbf{R}\left(\mathfrak{M}_{\widehat{\mathfrak{J}}}\right) \\
\left.\operatorname{Inv}\left(\mathfrak{M}_{\widehat{\mathfrak{J}}}\right)\right)
\end{gathered}
$$

This can be regarded as a consequence of the following Theorem by Dynkin (Th. 1.5 of [45], more recently discussed e.g. in [46]): every irreducible group of unimodular linear transformations of the $\mathrm{N}$-dimensional complex space (namely, a group of transformations which does not leave invariant a proper subspace of such a space) is maximal either in $\operatorname{SL}(N)$ (if the group does not have a bilinear invariant), or in $\operatorname{Sp}(N)$ (if it has a skew-symmetric bilinear invariant), or in $O(N)$ (if it has a symmetric bilinear invariant). Exceptions to this rule are listed in table VII of [46].

For later convenience, we introduce the number $f$ as (cfr. (2.4))

$$
\operatorname{dim}_{\mathbb{R}} \operatorname{Fund}(\mathfrak{s p}(\mathfrak{K}(\widehat{\mathfrak{J}})))=\operatorname{dim}_{\mathbb{R}} \mathbf{R}\left(\operatorname{Inv}\left(\mathfrak{M}_{\widehat{\mathfrak{J}}}\right)\right)=\operatorname{dim}_{\mathbb{R}} \mathfrak{K}(\widehat{\mathfrak{J}}) \equiv f
$$

which is even whenever the symplectic 2-form on $\mathfrak{K}(\widehat{\mathfrak{J}})$ is non-degenerate (as we will assume throughout).

From (2.3) and (2.5), it thus follows that the invariance subalgebra $\operatorname{Inv}\left(\mathfrak{M}_{\widehat{\mathfrak{J}}}\right)$ can be equivalently defined as the intersection of two Lie algebras: the symplectic one $\mathfrak{s p}(\mathfrak{K}(\widehat{\mathfrak{J}}))$ in $(2.5)$ and the exceptional one $\mathfrak{G}\left(=\mathfrak{g}_{2}, \mathfrak{f}_{4}, \mathfrak{e}_{6}, \mathfrak{e}_{7}, \mathfrak{e}_{8}\right)$ in $(2.3)$ :

$$
\operatorname{Inv}\left(\mathfrak{M}_{\widehat{J}}\right)=\mathfrak{s p}(\mathfrak{K}(\widehat{\mathfrak{J}})) \cap \mathfrak{G}
$$

\subsection{General case}

Within Freudenthal's formulation, the above construction can be repeated for a generic FTS $\mathfrak{K}$, by generalizing (2.2) to the submanifold

$$
\mathfrak{M}_{\mathfrak{J}} \equiv\left\{\phi_{I} \in \mathfrak{K}(\mathfrak{J}) \mid \mathcal{L}_{\phi_{I} \phi_{I}} \phi_{J}=0, \forall \phi_{J} \in \mathfrak{K}(\mathfrak{J})\right\}
$$

and thus introducing its invariance algebra $\operatorname{Inv}\left(\mathfrak{M}_{\mathfrak{J}}\right)$.

It is however worth remarking that, in this general case, neither $\operatorname{Inv}\left(\mathfrak{M}_{\mathfrak{J}}\right)$ nor

$$
\mathfrak{G}=\operatorname{Inv}\left(\mathfrak{M}_{\mathfrak{J}}\right) \oplus \mathfrak{s u}(2) \oplus \mathfrak{K}(\mathfrak{J}) \oplus \mathfrak{K}(\mathfrak{J})
$$

(this latter generalizing (2.3) to a generic JTS $\mathfrak{J}$ ), along with their possible non-compact real forms, are necessarily simple.

Nonetheless, it still holds that, as a vector space, $\mathfrak{K}(\mathfrak{J})$ may be regarded as the representation space of the relevant symplectic representation $\mathbf{R}$ of the invariance subalgebra $\operatorname{Inv}\left(\mathfrak{M}_{\mathfrak{J}}\right)$ of $\mathfrak{M}_{\mathfrak{J}}(2.8)$ :

$$
\mathfrak{K}(\mathfrak{J}) \sim \mathbf{R}\left(\operatorname{Inv}\left(\mathfrak{M}_{\mathfrak{J}}\right)\right)
$$


Before proceeding to analyze the axiomatic definition of FTS, we remark that, as mentioned in footnote 1 , in the mathematics literature there are several different notions of FTS, which differ by the symmetry structure of the corresponding triple product (see for instance $[22,41,47])$. All of these " $F T S$ 's" are closely inter-related by simple redefinitions; however, because they exhibit different symmetry properties, some algebraic properties of the FTS are manifest only within a specific formulation.

\subsection{Axiomatic definition}

We define an FTS to be a particular Symplectic Triple System [48, 49], which is a symplectic vector space $\mathfrak{K}$ equipped with a (not necessarily completely symmetric) triple product

$$
T:\left\{\begin{array}{l}
\mathfrak{K} \otimes \mathfrak{K} \otimes \mathfrak{K} \rightarrow \mathfrak{K} ; \\
\phi_{I}, \phi_{J}, \phi_{K} \mapsto T\left(\phi_{I}, \phi_{J}, \phi_{K}\right) .
\end{array}\right.
$$

In the following, for brevity's sake, we will denote $T\left(\phi_{I}, \phi_{J}, \phi_{K}\right) \equiv \phi_{I} \phi_{J} \phi_{K}$.

By introducing the symplectic form as ${ }^{5}$

$$
\langle\cdot, \cdot\rangle:\left\{\begin{array}{l}
\mathfrak{K} \otimes_{a} \mathfrak{K} \rightarrow \mathbb{R} ; \\
\phi_{I}, \phi_{J} \mapsto\left\langle\phi_{I}, \phi_{J}\right\rangle,
\end{array}\right.
$$

in an FTS the triple product (2.11) satisfies the following axioms:

(i) $\phi_{I} \phi_{J} \phi_{K}=\phi_{J} \phi_{I} \phi_{K}$

(ii) $\phi_{I} \phi_{J} \phi_{K}=\phi_{I} \phi_{K} \phi_{J}+2 \lambda\left\langle\phi_{J}, \phi_{K}\right\rangle \phi_{I}+\lambda\left\langle\phi_{I}, \phi_{K}\right\rangle \phi_{J}-\lambda\left\langle\phi_{I}, \phi_{J}\right\rangle \phi_{K}$

(iii) $\phi_{L} \phi_{M}\left(\phi_{I} \phi_{J} \phi_{K}\right)=\left(\phi_{L} \phi_{M} \phi_{I}\right) \phi_{J} \phi_{K}+\phi_{I}\left(\phi_{L} \phi_{M} \phi_{J}\right) \phi_{K}+\phi_{I} \phi_{J}\left(\phi_{L} \phi_{M} \phi_{K}\right)$;

(iv) $\left\langle\phi_{L} \phi_{M} \phi_{I}, \phi_{J}\right\rangle+\left\langle\phi_{I}, \phi_{L} \phi_{M} \phi_{J}\right\rangle=0$

where $\lambda$ is an arbitrary (real) constant. ${ }^{6}$

By introducing, for any pair $\phi_{L}, \phi_{M} \in \mathfrak{K}$, a linear operator $\mathcal{L}_{\phi_{L} \phi_{M}} \in \mathfrak{g l}(\mathfrak{K})$ acting on $\phi_{K} \in \mathfrak{K}$ as

$$
\mathcal{L}_{\phi_{I} \phi_{J}}:\left\{\begin{array}{l}
\mathfrak{K} \otimes_{s} \mathfrak{K} \rightarrow \mathfrak{K} ; \\
\phi_{I}, \phi_{J} \mapsto \mathcal{L}_{\phi_{I} \phi_{J}} \phi_{K} \equiv \phi_{I} \phi_{J} \phi_{K},
\end{array}\right.
$$

axiom (iii) yields that $\mathcal{L}_{\phi_{I} \phi_{J}}$ is a derivation with respect to the FTS triple product $T$ (2.11).

\footnotetext{
${ }^{5}$ Subscripts " $s$ " and " $a$ " respectively stand for symmetric and antisymmetric.

${ }^{6}$ Axioms (i)-(iv) define the most general FTS $\mathfrak{K}$, which does not necessarily enjoys the decomposition (2.1) in terms of an underlying JTS $\mathfrak{J}$ (as in the original Freudenthal's construction).

A counterexample is provided by Example 1 of [41], in which $\mathfrak{g}=\mathfrak{s p}(2 l)$. In $\mathcal{N}=1, D=4$ supergravity, this corresponds to a theory in which the scalar fields parametrize the upper Siegel half-plane; see e.g. a recent treatment in [53].
} 
On the other hand, axiom (i) implies

$$
\mathcal{L}_{\phi_{I} \phi_{J}}=\mathcal{L}_{\phi_{J} \phi_{I}}
$$

which justifies the symmetric tensor product of $\mathfrak{K}$ 's in the definition (2.13) itself.

By virtue of the definition (2.13), one can reformulate axioms (iii) and (iv) as follows:

$\left(i i i^{\prime}\right) \mathcal{L}_{\phi_{L} \phi_{M}}\left(\phi_{I} \phi_{J} \phi_{K}\right)=\left(\mathcal{L}_{\phi_{L} \phi_{M}} \phi_{I}\right) \phi_{J} \phi_{K}+\phi_{I}\left(\mathcal{L}_{\phi_{L} \phi_{M}} \phi_{J}\right) \phi_{K}+\phi_{I} \phi_{J}\left(\mathcal{L}_{\phi_{L} \phi_{M}} \phi_{K}\right) ;$

$\left(i v^{\prime}\right) \mathcal{L}_{\phi_{L} \phi_{M}}\left\langle\phi_{I}, \phi_{J}\right\rangle=\left\langle\mathcal{L}_{\phi_{L} \phi_{M}} \phi_{I}, \phi_{J}\right\rangle+\left\langle\phi_{I}, \mathcal{L}_{\phi_{L} \phi_{M}} \phi_{J}\right\rangle=0$.

In particular, the reformulation $\left(i v^{\prime}\right)$ of axiom $(i v)$ makes manifest the fact the symplectic form $\langle\cdot, \cdot\rangle(2.12)$ is invariant under $\mathcal{L}_{\phi_{I} \phi_{J}}$. Thus, $\mathcal{L}_{\phi_{I} \phi_{J}}$ is valued in a certain Lie algebra $\mathfrak{g}$, which exhibits a symplectic bilinear invariant structure in the relevant representation $\mathbf{R}$ to which $\phi_{I}$ belongs. At least when such a representation space is irreducible, through the GZ embedding [44], or equivalently through the abovementioned Dynkin Theorem [45]

$$
\mathfrak{g} \stackrel{G Z}{\subset} \mathfrak{s p}(\mathfrak{K}) \subset \mathfrak{g l}(\mathfrak{K}): \mathbf{R}(\mathfrak{g})=\text { Fund }(\mathfrak{s p})=\text { Fund }(\mathfrak{g l}),
$$

one has

$$
\mathcal{L}_{\phi_{I} \phi_{J}} \in \mathfrak{g} \stackrel{G Z}{\complement} \mathfrak{s p}(\mathfrak{K}) \subset \mathfrak{g l}(\mathfrak{K}) .
$$

Within Freudenthal's construction, an important class of algebras is given by $\mathfrak{g}=\operatorname{Inv}\left(\mathfrak{M}_{\widehat{\mathfrak{J}}}\right)$ introduced above. The Lie algebra $\mathfrak{g}$ will be identified below as the gauge Lie algebra of the Freudenthal gauge theory.

It is worth remarking here that for $\lambda \neq 0$ axiom (iv) can actually be derived from axioms (i)-(iii). Mathematically, whenever $\lambda \neq 0$ axiom (ii) yields a compatibility condition that constrains the structure of the triple product (2.11) and the symplectic form (2.12), and hence the non-trivial algebraic structure of the FTS itself. We anticipate that axiom (iii) can be regarded as the "FTS counterpart" of the so-called "fundamental identity" of Lie-3 algebras (see section 5). On the other hand, for $\lambda=0$ axioms (i)-(iii) reduce to the defining properties of a Lie-3 algebra over Grassmannian numbers, which in general is not a FTS. And hence, in order to restore the algebraic structure of the FTS $\mathfrak{K}$, one has to further impose axiom (iv) as a compatibility condition between the (now totally symmetric) triple product (2.11) and the symplectic form (2.12).

At any rate, in the present investigation we regard an FTS $\mathfrak{K}$ as a Symplectic Triple System $[48,49]$ with $\lambda \neq 0$, and we include (iv) (or equivalently $\left(i v^{\prime}\right)$ ) as part of the defining axioms, so that the most generic situation will be considered.

\section{$2.4 \quad F T S$ structure constants and their invariance}

In order to make our treatment more explicit yet basis-dependent, it is convenient to introduce a basis $\left\{e_{a}\right\}$ of $\mathfrak{K}$, such that $\phi=\phi^{a} e_{a}\left(a=1, \ldots, f ; f=\operatorname{dim}_{\mathbb{R}}(\mathfrak{K}),(2.6)\right)$. Thus, one can define the symplectic metric $\omega_{a b}$ and the FTS (triple product) structure constants $f_{a b c}{ }^{d}$ respectively as

$$
\left\langle e_{a}, e_{b}\right\rangle \equiv \omega_{a b}=-\omega_{b a} ;
$$




$$
e_{a} e_{b} e_{c} \equiv f_{a b c}{ }^{d} e_{d}
$$

As mentioned above, $\omega_{a b}$ is invariant under $\mathfrak{g}$ (recall (2.15) and (2.16)). Furthermore, when $\omega_{a b}$ is non-degenerate (which we will always assume to hold true in this paper), an isomorphism is defined between the vector space $\mathfrak{K}$ and its dual space, and hence one can lower ${ }^{7}$ the last index of the FTS structure constants as follows:

$$
f_{a b c d} \equiv f_{a b c}{ }^{e} \omega_{e d}
$$

By virtue of definitions (2.17), the defining axioms $(i)-(i v)$ of the FTS $\mathfrak{K}$ can be rewritten as follows:

(i) $f_{a b c d}=f_{b a c d}$;

(ii) $f_{a b c d}=f_{a c b d}+2 \lambda \omega_{a d} \omega_{b c}-\lambda \omega_{c a} \omega_{b d}-\lambda \omega_{a b} \omega_{c d}$;

(iii) $f_{a b c}{ }^{d} f_{\text {efd }}^{g}=f_{e f c}{ }^{d} f_{a b d}^{g}+f_{\text {ecf }}{ }^{d} f_{a d b}^{g}+f_{f c e}{ }^{d} f_{b d a}{ }^{g}$;

(iv) $f_{a b c d}=f_{a b d c}$.

It is worth stressing here that the non-complete symmetry of the FTS triple product $T$ (2.11) (as yielded by axioms $(i)$ and $(i i)$ ) implies the non-complete symmetry of the rank-4 tensor of FTS structure constants $f_{a b c d}(2.18)$. However, note that axioms $(i),(i i)$, and $(i v)$ imply the structure constants to be symmetric also under exchange of the first and last pair of its indices:

$$
f_{a b c d}=f_{c d a b},
$$

a property which will be important in the construction of a Chern-Simons action for the gauge fields of the "Freudenthal gauge theory" (see next sections).

Summarizing, the general symmetry properties of $f_{a b c d}$, as implied by axioms $(i),(i i)$ and $(i v)$, are given by

$$
f_{a b c d}=f_{((a b),(c d))} .
$$

$f_{a b c}{ }^{d}$ and $f_{a b c d}$ are rank-4 invariant tensors of the Lie algebra $\mathfrak{g}(2.15)-(2.16)$. Under certain further restrictions (see point 2 in section 5 ), the symmetry can be extended to $\mathfrak{s p}(\mathfrak{K})$ itself. It is here worth recalling that Kantor gave a complete classification of the finite dimensional triple systems that can arise in Lie algebras [50] (see also [51]); in particular, Kantor and Skopets showed that there is a one-to-one correspondence between simple Lie algebras and simple FTS's with a non-degenerate bilinear form [52].

\subsection{Freudenthal duality}

Whenever the completely symmetric part of $f_{a b c d}$ is non-vanishing, from the definition of the FTS triple product (2.11) and of the symplectic form (2.12) one can define a quartic

\footnotetext{
${ }^{7}$ We adopt the NE-WS convention when raising or lowering the indices using the symplectic metric.
} 
$\mathfrak{g}$-invariant structure $\Delta(\phi)$ for any $\phi \in \mathfrak{K}$, as follows ${ }^{8}$ (cfr. (25c) of [23]; $\left.T(\phi) \equiv \phi \phi \phi\right)$ :

$$
\Delta:\left\{\begin{array}{l}
\mathfrak{K} \rightarrow \mathbb{R} ; \\
\phi \mapsto \Delta(\phi) \equiv \frac{1}{2}\langle\phi \phi \phi, \phi\rangle=\frac{1}{2} f_{a b c d} \phi^{a} \phi^{b} \phi^{c} \phi^{d} .
\end{array}\right.
$$

Such a quartic form has appeared in physical literature e.g. in the formula for the Bekenstein-Hawking [32, 33] entropy of spherically symmetric, asymptotically flat, static, extremal black hole solutions of $D=4$ supergravity theories whose $U$-duality Lie algebra is a particular non-compact, real form of $\operatorname{Inv}\left(\mathfrak{M}_{\widehat{\mathfrak{J}}}\right)$, namely the conformal Lie algebra $\mathfrak{g}=\operatorname{conf}(\widehat{\mathfrak{J}})$ of $\widehat{\mathfrak{J}}$ itself (see e.g. [19] and [54] for a review, and a list of refs.).

Interestingly, $\Delta$ also occurs in the duality-invariant expression of the cosmological constant of some $A d S_{4}$ vacua (and of the corresponding central charge of the dual CFT's) of general $\mathcal{N}=2$ gauged supergravities underlying flux compactifications of type $I I$ theories [79].

The fact that $f_{(a b c d)} \neq 0$ which allows for the existence of (primitive) quartic $\mathfrak{g}$-invariant structure $\Delta(\phi)$ characterizes the pair $(\mathfrak{g}=\mathfrak{c o n} \mathfrak{f}(\widehat{\mathfrak{J}}), \mathbf{R})$ as a (non-degenerate) Lie algebra of type $\mathfrak{e}_{7}$, defined axiomatically by the axioms $(a)-(c)$ of [22]: $\mathbf{R}$ is a representation space of $\mathfrak{g}$ such that

(a) $\mathbf{R}$ possesses a non-degenerate, skew-symmetric bilinear $\mathfrak{g}$-invariant form (cfr. (2.12) and $(2.17))$;

(b) $\mathbf{R}$ possesses a completely symmetric, rank-4 $\mathfrak{g}$-invariant structure $f_{(a b c d)}$ ( given by the completely symmetric part of (2.18)), which allows to define

$$
q(x, y, z, w) \equiv f_{(a b c d)} x^{a} y^{b} z^{c} w^{d}=2 \Delta(x, y, z, w)
$$

(c) by defining a ternary product $\mathbf{T}(x, y, z)$ on $\mathbf{R}$ as

$$
\langle\mathbf{T}(x, y, z), w\rangle \equiv q(x, y, z, w)
$$

then one has

$$
3\langle\mathbf{T}(x, x, y), \mathbf{T}(y, y, y)\rangle=\langle x, y\rangle q(x, y, y, y) .
$$

Note that, from (2.22) and (2.23), $\mathbf{T}(x, y, z)$ is the the completely symmetric part of the triple product $T(2.11)$ on $\mathfrak{K} \sim \mathbf{R}$.

Recently, the role of Lie algebras of type $\mathfrak{e}_{7}$ was investigated in supergravity in some detail (see section 3.5). In section 5 Brown's definition of Lie algebras of type $\mathfrak{e}_{7}[22]$ will be discussed in relation to FTS and Freudenthal gauge theory.

\footnotetext{
${ }^{8}$ Even if here $f_{a b c d}$ is not (necessarily) completely symmetric in the present framework, we adopt the same normalization of [23] and [24].
} 
From the FTS axioms discussed in subsections 2.3 and 2.4, one can show that $\Delta(\phi)$ is invariant under the following transformation:

$$
\mathcal{F}:\left\{\begin{array}{l}
\mathfrak{K} \rightarrow \mathfrak{K} ; \\
\phi \mapsto \mathcal{F}(\phi) \equiv \operatorname{sgn}(\Delta(\phi)) \frac{T(\phi)}{\sqrt{6|\lambda \Delta(\phi)|}} \equiv \tilde{\phi}
\end{array}\right.
$$

namely that

$$
\Delta(\phi)=\Delta(\tilde{\phi}),
$$

The proof can be found in appendix A (which generalizes the treatment of [23], in turn referring to [22], to FTS defined by axioms $(i)-(i v)$; see also [24]). In the physics literature, the map $\mathcal{F}(2.25)$ has been called "Freudenthal Duality" (or F-duality for short); it was first observed in [23] as a symmetry of the Bekenstein-Hawking [32, 33] entropy-area formula for black holes, and then further generalized ${ }^{9}$ in [24].

In the rest of this subsection, we list some brief remarks; further details will be reported in a forthcoming paper [34].

(I) Anti-involutivity. The $F$-duality $\mathcal{F}(2.25)$ is an anti-involution in $\mathfrak{K}$ [22-24]:

$$
\begin{aligned}
\mathcal{F} \circ \mathcal{F} & =-I d ; \\
\tilde{\tilde{\phi}} & =-\phi .
\end{aligned}
$$

This holds whenever $\phi$ is an element in $\mathfrak{M}_{\mathfrak{J}}^{c}$, which is the complement in $\mathfrak{K}$ of the submanifold (recall (2.8))

$$
\left.\mathfrak{M}_{\mathfrak{J}}\right|_{I=J} \equiv\left\{\phi \in \mathfrak{K} \mid \mathcal{L}_{\phi \phi} \phi \equiv T(\phi)=0\right\} \subset \mathfrak{K} .
$$

In addition to this, for $\lambda \neq 0$ and for any $\phi \in \mathfrak{K}$, the $F$-duality map and its image $\tilde{\phi}$ (namely, the "F-dual" scalar field) are defined iff $\Delta(\phi) \neq 0$. Whenever $\operatorname{Inv}\left(\mathfrak{M}_{\mathfrak{J}}\right)$ is non-empty and thus its corresponding action determines a stratification of the symplectic vector space $\mathfrak{K}(\mathfrak{J}) \sim \mathbf{R}\left(\operatorname{Inv}\left(\mathfrak{M}_{\mathfrak{J}}\right)\right)$ (cfr. (2.10)), this can also be equivalently stated as the requirement that $\phi$ belongs to the rank- 4 orbit of $\mathfrak{K}$ under the action of $\operatorname{Inv}\left(\mathfrak{M}_{\mathfrak{J}}\right)$ itself.

(II) $\mathbb{Z}_{4}$-grading. The anti-involutivity $(2.27)$ of $\mathcal{F}$ yields a $\mathbb{Z}_{4}$-grading of the symplectic vector space $\mathfrak{K}$. This interesting property will be investigated in [34].

(III) $\boldsymbol{F}$-duality is not an $\boldsymbol{F T S}$ derivation. The non-linear map over $\mathfrak{K}$ provided by F-duality (2.25) is not a derivation with respect to the triple product (2.11) over $\mathfrak{K}$. Thus, such a mathematical structure cannot be consistently used to define an infinitesimal transformation. This means that the invariance (2.26) is rather a global symmetry ("duality") of $\mathfrak{K}$, and thus a global (off-shell) symmetry of the corresponding gauge theory; see next sections.

\footnotetext{
${ }^{9}$ In the nomenclature introduced in [24], (2.25) (which preserves the homogeneity in $\phi$ ) defines the nonpolynomial "on-shell" version of F-duality; other possible versions and generalizations are discussed therein.
} 


\section{Freudenthal Gauge Theory (FGT)}

In the present section, we will introduce the gauge theory based on the FTS discussed in section 2. As anticipated, this theory, whose consistent (bosonic) Lagrangian density is proposed in subsection 3.3, will be named "Freudenthal Gauge Theory" (FGT).

As it will become clear, our construction resembles very much the one of BLG theory $[10,11]$. However, we present here a detailed analysis, also in order to make several remarks addressing the differences between FGT (and thus FTS) and the triple systemsrelated gauge theories, especially in $D=3$ (see the discussion in section 5).

\subsection{From global symmetry...}

We consider a real scalar field $\phi(x)$ valued in a FTS $\mathfrak{K}$ over $\mathbb{R}$, and we aim at constructing a Lagrangian density functional $\mathbf{L}[\phi(x)]$ with the desired symmetry.

Clearly, $\mathbf{L}[\phi(x)]$ must be a $\mathfrak{K}$-scalar, and thus all its terms must be of the form

$$
\begin{aligned}
& \mathbf{L}[\phi(x)] \sim \alpha(\phi)\langle f(\phi), g(\phi)\rangle, \\
& \alpha:\left\{\begin{array}{l}
\mathfrak{K} \rightarrow \mathbb{R} ; \\
\phi(x) \mapsto \alpha(\phi(x)) ;
\end{array}\right. \\
& f, g:\left\{\begin{array}{l}
\mathfrak{K} \rightarrow \mathfrak{K} ; \\
\phi(x) \mapsto f(\phi(x)) ; \phi(x) \mapsto g(\phi(x)) .
\end{array}\right.
\end{aligned}
$$

At each point $x$ in space-time, $f(\phi(x))$ and $g(\phi(x))$ are elements of the subalgebra $\mathfrak{K}_{\phi(x)} \subset$ $\mathfrak{K}$ generated by the element $\phi(x) \in \mathfrak{K}$. More precisely, elements of $\mathfrak{K}_{\phi(x)}$ are homogeneous polynomials of odd degree in $\phi(x)$, with the multiplication defined by the non-associative (cfr. axiom (iii)) triple product $T(2.11)$ over $\mathfrak{K}$.

The FTS axiom (iii) (or equivalently $\left(i i^{\prime}\right)$ ), along with the definition (2.13), allow for a consistent definition of an infinitesimal transformation $\mathcal{L}_{\Lambda} \in \mathfrak{s p}(\mathfrak{K})$ (recall (2.16)), such that

$$
\left[f\left(\left(I d+\mathcal{L}_{\Lambda}\right) \phi(x)\right)-f(\phi(x))\right]_{\text {linear order }}=\mathcal{L}_{\Lambda} f(\phi(x)),
$$

where the parameters of the transformation are denoted by

$$
\Lambda \in \mathfrak{K} \otimes_{s} \mathfrak{K} .
$$

Note that only elements in the symmetric tensor product $\mathfrak{K} \otimes_{s} \mathfrak{K}$ can generate a transformation $\mathcal{L}_{\Lambda}$, because the antisymmetric part $\mathfrak{K} \otimes_{a} \mathfrak{K}$ is projected out by the symmetry property under the exchange of the first two entries of the triple product $T$ (cfr. axiom $(i)$ ).

Crucially, axiom (iv) (or equivalently $\left(i v^{\prime}\right)$ ) states that for any $f(\phi), g(\phi) \in \mathfrak{K}$, the symplectic product $\langle f(\phi), g(\phi)\rangle$ (defined in (2.12) and in $(2.17)$ ) is invariant under $\mathcal{L}_{\Lambda}$ :

$$
\mathcal{L}_{\Lambda}\langle f(\phi), g(\phi)\rangle=\left\langle\mathcal{L}_{\Lambda} f(\phi), g(\phi)\right\rangle+\left\langle f(\phi), \mathcal{L}_{\Lambda} g(\phi)\right\rangle=0 .
$$

By the same argument, all $\mathfrak{K}$-scalar real functions $\alpha(\phi)(3.2)$ are necessarily of this form, namely

$$
\alpha(\phi) \sim\langle h(\phi), l(\phi)\rangle
$$

for some functions $h(\phi)$ and $l(\phi)$ of the same kind as $f(\phi)$ and $g(\phi)$ defined in (3.3). 
Thus, one can conclude that any Lagrangian density functional $\mathbf{L}$ of the form (3.1) is invariant $^{10}$ under the infinitesimal transformation (3.4). In other words, by the four axioms (i)-(iv) of FTS, any Lagrangian $\mathbf{L}$ of the form (3.1) is guaranteed to be invariant under the global symmetry generated by $\mathcal{L}_{\Lambda}(3.4)$.

It should also be remarked here that the definitions (2.21) and (2.25) imply that the $F$-dual field $\tilde{\phi}(x)$ is also an element of $\mathfrak{K}_{\phi(x)}$. Therefore, $\tilde{\phi}(x)$ transforms in the very same way as $\phi(x)$ under the global symmetry $\mathcal{L}_{\Lambda}(3.4)$.

As already pointed out above, the invariance (3.6) of the symplectic product $\langle\cdot, \cdot\rangle(2.12)$ in $\mathfrak{K}$ under the action of the infinitesimal transformation $\mathcal{L}_{\Lambda}$ implies that the latter is not simply an element in $\mathfrak{g l}(\mathfrak{K})$, but rather it generally belongs to the Lie algebra $\mathfrak{g}(2.15)-(2.16)$.

\section{$3.2 \quad$...to gauge symmetry}

We will now proceed to gauge the global symmetry introduced in subsection 3.1, by promoting the infinitesimal generator $\Lambda$ (3.5) to be a function $\Lambda(x)$ over space-time. Correspondingly, this will identify $\mathfrak{g}(2.15)-(2.16)$ as the gauge algebra.

As done in subsection 2.3 , by adopting a basis $\left\{e_{a}\right\}$ for $\mathfrak{K}$, one can generally write down the gauge transformation of a $\mathfrak{K}$-valued scalar field $\phi(x)=\phi^{a}(x) e_{a}$ in the following form (recall (2.17)):

$$
\mathcal{L}_{\Lambda} \phi(x)=\Lambda^{a b}(x) \mathcal{L}_{e_{a} e_{b}} \phi(x)=f_{a b c}{ }^{d} \Lambda^{a b}(x) \phi^{c}(x) e_{d},
$$

where $\Lambda^{a b}(x)$ denotes the rank-2 tensor generating the gauge transformation itself. Note that axiom (i) of FTS implies that such a tensor is symmetric (cfr. (2.14)):

$$
\mathcal{L}_{e_{a} e_{b}}=\mathcal{L}_{e_{b} e_{a}} \Leftrightarrow \Lambda^{a b}(x)=\Lambda^{b a}(x),
$$

which is consistent with (3.5). When $\Lambda^{a b}$ is constant over space-time, one consistently re-obtains the global symmetry considered in subsection 3.1 .

By recalling (2.16), one can define the linear operator $\hat{\Lambda} \in \mathfrak{g}$ as ${ }^{11}$

$$
\widehat{\Lambda}_{b}^{a} \equiv f_{c d b}{ }^{a} \Lambda^{c d}
$$

such that the gauge symmetry transformation (3.8) of a field $\phi(x)$ is nothing but a matrix multiplication by the linear operator $\hat{\Lambda}$ :

$$
\mathcal{L}_{\Lambda} \phi^{a}=\widehat{\Lambda}_{b}^{a} \phi^{b}
$$

As discussed at the end of subsection 3.1, the gauge transformation of the F-dual field $\tilde{\phi}(x)(2.25)$ is by construction the following one:

$$
\mathcal{L}_{\Lambda} \tilde{\phi}^{a}=\widehat{\Lambda}_{b}^{a} \tilde{\phi}^{b}
$$

\footnotetext{
${ }^{10}$ Note that no mentioning of invariance under (global; cfr. point (IV) of subsection 2.5) Freudenthal duality $\mathcal{F}$ (2.25) (which will be a crucial ingredient of FGT; see subsection 3.3) has been made so far; indeed, it is immediate to check that the Lagrangian density functional $\mathbf{L}$ (3.1) is not invariant under $\mathcal{F}(2.25)$.

${ }^{11}$ In the following treatment, we will often drop the explicit $x$-dependence in order to simplify the notation, whenever confusion is unlikely to occur.
} 
Next, we introduce a gauge field

$$
A_{\mu}(x) \equiv A_{\mu}^{a b}(x) e_{a} \otimes_{s} e_{b},
$$

which is a 1 -form valued $\operatorname{in}^{12} \mathfrak{K} \otimes_{s} \mathfrak{K}$. Correspondingly, a $\mathfrak{g}$-valued gauge covariant derivative $D_{\mu}$ acting on the scalar field $\phi^{a}(x)$ can be defined as:

$$
D_{\mu} \phi^{a}(x) \equiv \partial_{\mu} \phi^{a}(x)-\left(\widehat{A}_{\mu}\right)_{b}{ }^{a}(x) \phi^{b}(x),
$$

where

$$
\left(\widehat{A}_{\mu}\right)_{b}{ }^{a}(x) \equiv f_{c d b}{ }^{a} A_{\mu}^{c d}(x)
$$

is the corresponding 1-form linear operator in $\mathfrak{g}$.

It is worth remarking that both definitions (3.10) and (3.15) can respectively be regarded as images of the rank-2 symmetric tensor $\Lambda^{a b}(x)$ (3.5) of infinitesimal gauge parameters and of the corresponding rank-2 symmetric tensor $A_{\mu}^{a b}(x)$ (3.13) of 1-form gauge potentials, under a map (dubbed "hat" map), defined through the FTS structure constants $f_{a b c}{ }^{d}(2.17)$ as follows:

$$
\widehat{\cdot}:\left\{\begin{array}{l}
\mathfrak{K} \otimes_{s} \mathfrak{K} \rightarrow \mathfrak{g} ; \\
\Psi^{a b}(x) e_{a} \otimes_{s} e_{b} \mapsto f_{c d b}{ }^{a} \Psi^{c d}(x) \equiv \widehat{\Psi}_{b}^{a} .
\end{array}\right.
$$

The "hat" map (3.16) allows one to implement (generally g-valued) infinitesimal gauge transformation $\mathcal{L}_{\Lambda}$ defined via the FTS triple product in terms of standard matrix multiplication (in $\mathfrak{g l}(\mathfrak{K}))$. As such, this map provides an explicit matrix realization of the gauge Lie algebra $\mathfrak{g}$ of the FGT, by means of an embedding (local in space-time) analogous to the local embedding $\mathfrak{K}_{\phi(x)} \subset \mathfrak{K}$ mentioned below (3.3).

Then, the requirement of $D_{\mu} \phi(x)$ to transform under the gauge symmetry $\mathcal{L}_{\Lambda}$ in the same way as $\phi(x)$, i.e.

$$
\mathcal{L}_{\Lambda}\left(D_{\mu} \phi^{a}(x)\right)=\left(\mathcal{L}_{\Lambda} D_{\mu}\right) \phi^{a}(x)+D_{\mu}\left(\mathcal{L}_{\Lambda} \phi\right)^{a}(x) \equiv \widehat{\Lambda}_{b}{ }^{a}(x)\left(D_{\mu} \phi\right)^{b}(x)
$$

consistently fixes the gauge transformation $\widehat{A}_{\mu}(x)$ as follows:

$$
\mathcal{L}_{\Lambda} \widehat{A}_{\mu}(x)=\partial_{\mu} \widehat{\Lambda}(x)-\left[\widehat{A}_{\mu}(x), \widehat{\Lambda}(x)\right] \equiv D_{\mu} \widehat{\Lambda}(x),
$$

namely $\widehat{A}_{\mu}(x)$ transforms as a $\mathfrak{g}$-valued 1 -form.

To proceed further, we introduce the gauge field strength 2 -form

$$
\widehat{F}_{\mu \nu} \equiv-\left[D_{\mu}, D_{\nu}\right]=\partial_{\mu} \widehat{A}_{\nu}-\partial_{\nu} \widehat{A}_{\mu}-\left[\widehat{A}_{\mu}, \widehat{A}_{\nu}\right] \in \mathfrak{g},
$$

whose infinitesimal gauge transformation can consistently be computed to be

$$
\mathcal{L}_{\Lambda} \widehat{F}_{\mu \nu}=\left[\widehat{F}_{\mu \nu}, \widehat{\Lambda}\right] .
$$

The matrix embedding of $\mathcal{L}_{\Lambda}$ into $\mathfrak{g}$ provided by the "hat" map (3.16) also ensures that the "trace" of the field strength $\widehat{F}_{\mu \nu}(x)(3.19)$ is $\mathfrak{g}$-gauge invariant; in the next subsection, this fact will be used to work out a bosonic Lagrangian for FGT.

\footnotetext{
${ }^{12}$ Note that the symmetric nature of the tensor product in (3.13) does not imply any loss of generality, due to the axiom $(i)$ of FTS (yielding $f_{c d b}{ }^{a}=f_{(c d) b}{ }^{a}$ ).
} 


\subsection{The Lagrangian}

We are now going to propose a consistent bosonic Lagrangian for the FGT.

By recalling definitions (2.21) and (2.25) and considering the lowest possible order in the scalar field $\phi(x)$, one can introduce the following (generally non-polynomial) term

$$
\langle\phi, \tilde{\phi}\rangle=\operatorname{sgn}(\Delta(\phi)) \frac{\langle\phi, T(\phi)\rangle}{\sqrt{6|\lambda \Delta(\phi)|}}=-\sqrt{\frac{2}{3|\lambda|}} \sqrt{|\Delta(\phi)|},
$$

which is homogeneous of degree 2 in $\phi(x)$. As discussed in subsection 3.2, the gauge covariant derivatives of both $\phi(x)$ and its $F$-dual field $\tilde{\phi}(x)$ transform as vectors under the gauge transformation $\mathcal{L}_{\Lambda}$; therefore, a consistent kinetic term for scalar fields reads

$$
-\frac{1}{2}\left\langle D_{\mu} \phi, D^{\mu} \tilde{\phi}\right\rangle
$$

whose gauge invariance is guaranteed by the FTS axioms $(i)-(i v),(3.6)$, and by the very treatment of subsection 3.2.

From axiom (iv) (or equivalently (3.6)) and (3.21), it follows that for any sufficiently smooth function $V: \mathbb{R} \rightarrow \mathbb{R}$, then ${ }^{13}$

$$
V(\Delta(\phi))
$$

is a gauge invariant real function of $\phi$ :

$$
\mathcal{L}_{\Lambda}(V(\Delta(\phi)))=0
$$

which therefore can be taken as a gauge invariant potential in the bosonic FGT action.

By exploiting the matrix embedding of $\mathfrak{g}$-valued Freudenthal gauge transformations $\mathcal{L}_{\Lambda}$ (realized by the "hat" map (3.16)), one can construct a Maxwell term for the gauge invariant kinetic term for the gauge field $\hat{A}_{\mu}(x)$.

By introducing the Minkowski metric $\eta_{\mu \nu}=\eta^{\mu \nu}$ and a function $\mathcal{N}(\Delta(\phi))$ coupling vector and scalar fields, for $D \geqslant 4$ the following kinetic Maxwell term can be constructed:

$$
\begin{aligned}
\frac{1}{4} \mathcal{N}(\Delta(\phi)) \operatorname{Tr}\left(\widehat{F}^{2}\right) & \equiv \frac{1}{4} \mathcal{N}(\Delta(\phi))\left(\widehat{F}_{\mu \nu}\right)_{a}^{b}\left(\widehat{F}^{\mu \nu}\right)_{b}{ }^{a} \\
& =\frac{1}{4} \mathcal{N}(\Delta(\phi)) \eta^{\mu \lambda} \eta^{\nu \rho} f_{c d a}{ }^{b} f_{\text {efb }}{ }^{a} F_{\mu \nu}^{c d} F_{\lambda \rho}^{e f} \\
& =-\frac{1}{4} \mathcal{N}(\Delta(\phi)) \eta^{\mu \lambda} \eta^{\nu \rho} f_{c d a g} f_{e f b h} \omega^{a h} \omega^{g b} F_{\mu \nu}^{c d} F_{\lambda \rho}^{e f} .
\end{aligned}
$$

The gauge invariance of (3.25) results from the simple computation

$$
\begin{aligned}
\mathcal{L}_{\Lambda}\left(\frac{1}{4} \mathcal{N}(\Delta(\phi)) \operatorname{Tr}\left(\widehat{F}^{2}\right)\right) & =\frac{1}{4} \mathcal{L}_{\Lambda}(\mathcal{N}(\Delta(\phi))) \operatorname{Tr}\left(\widehat{F}^{2}\right)+\frac{1}{4} \mathcal{N}(\Delta(\phi)) \mathcal{L}_{\Lambda}\left(\operatorname{Tr}\left(\widehat{F}^{2}\right)\right)(3.2 \\
& =\frac{1}{2} \mathcal{N}(\Delta(\phi)) \operatorname{Tr}([\widehat{F}, \widehat{\Lambda}] \widehat{F})=0
\end{aligned}
$$

\footnotetext{
${ }^{13}$ Actually, by recalling definitions (3.2) and (3.3), one could have chosen $V(\alpha(\phi)\langle f(\phi), g(\phi)\rangle)$ as the most general gauge invariant potential term. However, the invariance also under F-duality $\mathcal{F}(2.25)$, as we do impose in FGT (see further below), further restricts the choice to $V(\Delta(\phi))$, as given by (3.23).
} 
where (3.24) has been used for the function $\mathcal{N}$, the field strength gauge transformation property (3.20) has been recalled, and the cyclicity of the trace has been exploited.

Thus, by merging (3.22), (3.23) and (3.25), the following (bosonic) Lagrangian for the "Freudenthal gauge theory"(FGT) can be written down:

$$
\mathbf{L}\left[\phi(x), F_{\mu \nu}(x)\right]_{D \geqslant 4}=-\frac{1}{2}\left\langle D_{\mu} \phi, D^{\mu} \tilde{\phi}\right\rangle+\frac{1}{4} \mathcal{N}(\Delta(\phi)) \operatorname{Tr}\left(\widehat{F}^{2}\right)-V(\Delta(\phi)),
$$

whose simplest ( "minimal") version corresponds to setting $V(\Delta(\phi))=\Delta(\phi)$ (quartic scalar potential) and $\mathcal{N}(\Delta(\phi))=1$ :

$$
\mathbf{L}_{\text {minimal }}\left[\phi(x), F_{\mu \nu}(x)\right]_{D \geqslant 4}=-\frac{1}{2}\left\langle D_{\mu} \phi, D^{\mu} \tilde{\phi}\right\rangle+\frac{1}{4} \operatorname{Tr}\left(\widehat{F}^{2}\right)-\Delta(\phi) .
$$

Remarkably, the FGT Lagrangian density functional $\mathbf{L}\left[\phi(x), F_{\mu \nu}(x)\right]_{D \geqslant 4}(3.28)$ is not only invariant under the off-shell gauge Lie algebra $\mathfrak{g}$ introduced in subsections $3.1-3.2$, but also under the $F$-duality $\mathcal{F}(2.25)$, which acts as a global (off-shell) symmetry. ${ }^{14} \mathrm{In}$ order to check this, one should simply recall (2.26), as well as the anti-involutivity (2.27) of $\mathcal{F}(2.25)$ itself and the anti-symmetry of the symplectic product used to construct the scalar kinetic term (3.22). In particular, the $\mathcal{F}$-invariance of the latter reads (recall point (IV) of subsection 2.5):

$$
\begin{aligned}
\mathcal{F}\left(\eta^{\mu \nu}\left\langle D_{\mu} \phi, D_{\nu} \tilde{\phi}\right\rangle\right) & =\eta^{\mu \nu}\left\langle D_{\mu} \tilde{\phi}, D_{\nu}(-\phi)\right\rangle=\eta^{\mu \nu}\left\langle D_{\nu} \phi, D_{\mu} \tilde{\phi}\right\rangle \\
& =\eta^{\mu \nu}\left\langle D_{\mu} \phi, D_{\nu} \tilde{\phi}\right\rangle,
\end{aligned}
$$

where in the second line one does not necessarily have to use the the symmetry of the Minkowski space-time metric $\eta^{\mu \nu}$, because, the scalar kinetic term is symmetric under the exchange of its space-time indices:

$$
\left\langle D_{\mu} \phi, D_{\nu} \tilde{\phi}\right\rangle=\left\langle D_{\nu} \phi, D_{\mu} \tilde{\phi}\right\rangle,
$$

as shown in appendix B.

It should be remarked here that in the above construction the dimension $D$ of spacetime does not necessarily need to be specified. As mentioned, the ( $\phi$-coupled) Maxwell kinetic vector term (3.25) is well defined in $D \geqslant 4$. Moreover, in $D=4$ a topological (theta) term can also be introduced, along with its vector-scalar coupling function $\mathcal{M}(\Delta(\phi))$ :

$$
\frac{1}{4} \mathcal{M}(\Delta(\phi)) \operatorname{Tr}(\widehat{F} \wedge \widehat{F}),
$$

and its gauge invariance and $\mathcal{F}$-invariance once again follow from (3.24), (3.20), (2.26) and the the cyclicity of the trace.

Thus, in $D=4$, the bosonic Lagrangian density (3.28) can be completed as follows:

$$
\begin{aligned}
\mathbf{L}\left[\phi(x), F_{\mu \nu}(x)\right]_{D=4}= & -\frac{1}{2}\left\langle D_{\mu} \phi, D^{\mu} \tilde{\phi}\right\rangle-V(\Delta(\phi)) \\
& +\frac{1}{4} \mathcal{N}(\Delta(\phi)) \operatorname{Tr}\left(\widehat{F}^{2}\right)+\frac{1}{4} \mathcal{M}(\Delta(\phi)) \operatorname{Tr}(\widehat{F} \wedge \widehat{F}) .
\end{aligned}
$$

\footnotetext{
${ }^{14}$ From point $(\mathbf{I V})$ of subsection 2.5 , the Freudenthal duality $\mathcal{F}(2.25)$ is not a derivation with respect to the FTS triple product (2.11) over $\mathfrak{K}$, and thus with respect to the FTS-based gauge transformation introduced above.
} 
Even if in the above construction the dimension $D$ of space-time does not necessarily need to be specified, it should be stressed that in $D \geqslant 4$ the FGT is non-unitary whenever the gauge Lie algebra $\mathfrak{g}$ is non-compact (and thus with a Cartan-Killing metric which is not positive-definite). Indeed, we recall that in the present investigation we consider the FTS to be defined on the ground field $\mathbb{R}$ (cfr. footnote 1 ); this constrains the pair $(\mathfrak{g}, \mathbf{R}$ ) such that $\mathbf{R}$ is a real representation space of the real algebra $\mathfrak{g}$. The latter, at least in the examples related to conformal symmetries of JTS $\mathfrak{J}=\widehat{\mathfrak{J}}$ (treated in section 3.4 and reported in table 1), is non-compact.

On the other hand, in $D=3$ space-time dimensions this does not hold any more, and the non-compactness of the (real) gauge Lie algebra $\mathfrak{g}$ is not inconsistent with unitarity of the theory. Indeed, $\mathbf{R}$ is always assumed to possess a positive-definite inner product (for unitarity of the corresponding gauge theory), but the gauge fields are not propagating (and they are in $\operatorname{Adj}(\mathfrak{g})$ ), and therefore $\mathfrak{g}$ does not necessarily have to be endowed with a positive-definite product, thus allowing for non-compact (real) forms of $\mathfrak{g}$ itself. As we discuss in section 5, this is particularly relevant for the connection between $D=3$ FGT and (the bosonic sector of) superconformal Chern-Simons-matter gauge theories in $D=3$.

Moreover, in $D=3$ a Chern-Simons (CS) term for the gauge sector can be considered, with the same form as in the BLG theory (cfr. (45) of [10]):

$$
\frac{1}{2} \varepsilon^{\mu \nu \lambda}\left(f_{a b c d} A_{\mu}^{a b} \partial_{\nu} A_{\lambda}^{c d}+\frac{2}{3} f_{c d a}{ }^{g} f_{e f g b} A_{\mu}^{a b} A_{\nu}^{c d} A_{\lambda}^{e f}\right),
$$

whose consistence in FGT follows from FTS axioms $(i)$ and $(i v)$. The $\mathcal{F}$-invariance of the CS term (3.34) is trivial (it does not depend on $\phi$ at all), while its gauge invariance can be easily proved by exploiting the symmetry property (2.19) of FTS structure constants $f_{a b c d}$.

Thus, in $D=3$ one can propose the following bosonic FGT Lagrangian density:

$$
\begin{aligned}
\mathbf{L}\left[\phi(x), F_{\mu \nu}(x)\right]_{D=3}= & -\frac{1}{2}\left\langle D_{\mu} \phi, D^{\mu} \tilde{\phi}\right\rangle-V(\Delta(\phi)) \\
& +\frac{1}{2} \varepsilon^{\mu \nu \lambda}\left(f_{a b c d} A_{\mu}^{a b} \partial_{\nu} A_{\lambda}^{c d}+\frac{2}{3} f_{c d a}{ }^{g} f_{e f g b} A_{\mu}^{a b} A_{\nu}^{c d} A_{\lambda}^{e f}\right) .
\end{aligned}
$$

\subsection{Gauge algebras of type $\mathfrak{e}_{7}$}

An interesting class of gauge algebras $\mathfrak{g}(2.15)-(2.16)$ for the FGT can be obtained by considering symmetry algebras of Jordan algebras $\widehat{\mathfrak{J}}$ themselves. Indeed, a particular noncompact, real form of the decomposition (2.3) reads

$$
\mathfrak{q c o n f}(\widehat{\mathfrak{J}})=\operatorname{conf}(\widehat{\mathfrak{J}}) \oplus \mathfrak{s l}(2, \mathbb{R}) \oplus \mathfrak{K}(\widehat{\mathfrak{J}}) \oplus \mathfrak{K}(\widehat{\mathfrak{J}}),
$$

where $\operatorname{conf}(\widehat{\mathfrak{J}})$ and $\mathfrak{q c o n f}(\widehat{\mathfrak{J}})$ respectively denote the conformal and quasi-conformal ${ }^{15}$ Lie algebras of rank-3 simple Euclidean Jordan algebras $\widehat{\mathfrak{J}}$. Note that $\operatorname{con} \mathfrak{f}(\widehat{\mathfrak{J}})$ is nothing but

\footnotetext{
${ }^{15}$ The novel, non-linear geometric quasi-conformal realizations of groups were first discovered by Günaydin, Koepsell and Nicolai in [16], by exploiting the underlying FTS, and showing that they extend to the complex forms and hence to different real forms of the corresponding groups. In the subsequent papers [17] and [18], the quasi-conformal realizations of $D=3 U$-duality groups of Maxwell-Einstein supergravity theories, respectively with with 8 and at least 16 supersymmetries, have been determined. See e.g. [19], for a review and a list of refs..
} 
a particular non-compact, real form of $\operatorname{Inv}\left(\mathfrak{M}_{\widehat{J}}\right)$; this is also consistent with the fact that $\mathfrak{c o n f}(\widehat{\mathfrak{J}})$ is nothing but the automorphism Lie algebra of $\mathfrak{K}(\widehat{\mathfrak{J}})$ itself:

$$
\operatorname{conf}(\widehat{\mathfrak{J}}) \sim \mathfrak{a u t}(\mathfrak{K}(\widehat{\mathfrak{J}})) .
$$

Analogously, also formulæ (2.4)-(2.7) hold at the suitable non-compact real level, by respectively replacing $\operatorname{Inv}\left(\mathfrak{M}_{\widehat{\mathfrak{J}}}\right)$ and $\mathfrak{s p}(\mathfrak{K}(\widehat{\mathfrak{J}}))$ with $\operatorname{con} \mathfrak{f}(\widehat{\mathfrak{J}}) \operatorname{and}^{16} \mathfrak{s p}(f, \mathbb{R})$. In particular, $(2.7)$ can be recast as

$$
\operatorname{conf}(\widehat{\mathfrak{J}})=\mathfrak{s p}(f, \mathbb{R}) \cap \mathfrak{q c o n f}(\widehat{\widetilde{J}}) .
$$

The decompositions (2.3) and (3.36), as well as the whole treatment above, also hold for rank-3 semi-simple Euclidean Jordan algebras of the type

$$
\widehat{\mathfrak{J}}=\mathbb{R} \oplus \boldsymbol{\Gamma}_{m, n},
$$

where $\boldsymbol{\Gamma}_{m, n}$ is a rank-2 Jordan algebra with a quadratic form of pseudo-Euclidean signature $(m, n)$, i.e. the Clifford algebra of $O(m, n)[80]$. However, in this case the corresponding Lie algebra $\mathfrak{G}$ in $(2.3)$ (or $\mathfrak{q} c \mathfrak{n} \mathfrak{f}(\widehat{\mathfrak{J}})$ in $(3.36))$ is a classical Lie algebra, namely a (pseudo)orthogonal algebra.

Table 1 lists the entries of (3.36) for rank-3 Euclidean Jordan algebras, also including the cases $\widehat{\mathfrak{J}}=J_{3}^{\mathfrak{A}_{s}} \equiv H_{3}\left(\mathfrak{A}_{s}\right)$, where $\mathfrak{A}_{s}=\mathbb{C}_{s}, \mathbb{H}_{s}, \mathbb{O}_{s}$ are the split version of $\mathbb{C}, \mathbb{H}, \mathbb{O}$, respectively (see e.g. [19] for further elucidation and list of refs.). The role of $\mathfrak{K}(\widehat{\mathfrak{J}})$ 's and their symmetries in supergravity is discussed in the next subsection 3.5.

It is also worth recalling here that the Lie algebra $\operatorname{Inv}\left(\mathfrak{M}_{\widehat{\mathfrak{J}}}\right)$ (or equivalently $\operatorname{conf}(\widehat{\mathfrak{J}})$ ) is "of type $\mathfrak{e}_{7}$ " [22], as recalled in section 2.5, and in the mathematical literature its symplectic (real) representation $\mathbf{R}$ is sometimes called minuscule irrep. (see e.g. [55]).

\subsection{FGT and supergravity}

Summarizing, a class of gauge algebras (and representations) for FGT is provided by the conformal Lie algebras conf of (simple and semi-simple) Euclidean, rank-3 algebras $\widehat{\mathfrak{J}}$, listed in table 1, along with their (real) symplectic representation $\mathbf{R}$. The pair $(\operatorname{con} \mathfrak{f}(\widehat{\mathfrak{J}}), \mathbf{R})$ characterizes $\operatorname{con} \mathfrak{f}(\widehat{\mathfrak{J}})$ as a Lie algebra of type $\mathfrak{e}_{7}[22]$.

Interestingly, $\operatorname{con} \mathfrak{f}(\widehat{\mathfrak{J}})$ is the $U$-duality ${ }^{17}$ Lie algebra of $D=4$ Maxwell-Einstein supergravity theories (MESGT's) related to the FTS $\mathfrak{K}(\widehat{\mathfrak{J}})[14,15]$ (see also e.g. [19] and [20, 21] for recent reviews, and list of refs.).

Indeed, within such a class of theories, the decomposition (3.36) can be further interpreted as the Cartan decomposition of the $\mathfrak{q} \mathfrak{c o n f}(\widehat{\mathfrak{J}})(U$-duality algebra in $D=3)$ with respect to $\operatorname{conf}(\widehat{\mathfrak{J}})(U$-duality algebra in $D=4)$. In particular, $\mathbf{R}(\mathfrak{c o n f}(\widehat{\mathfrak{J}}))$ listed in table 1 is the representation in which the 2 -form field strengths of the $D=4$ Abelian vector

\footnotetext{
${ }^{16}$ Note that $\mathfrak{s p}(f, \mathbb{R})$ is the maximally non-compact $($ split) real form of $\mathfrak{s p}(\mathfrak{K}(\widehat{\mathfrak{J}}))$.

${ }^{17}$ Here $U$-duality is referred to as the "continuous" symmetries of $[29,30]$. Their discrete versions are the $U$-duality non-perturbative string theory symmetries introduced by Hull and Townsend [31].
} 


\begin{tabular}{|c|c|c|c|c|}
\hline$\widehat{\mathfrak{J}}$ & $\mathfrak{c o n f}(\widehat{\mathfrak{J}})$ & $\mathfrak{q c o n f}(\widehat{\mathfrak{J}})$ & $\mathbf{R}(\mathfrak{c o n f}(\widehat{\mathfrak{J}}))$ & $\mathcal{N}$ \\
\hline $\mathbb{R}$ & $\mathfrak{s l}(2, \mathbb{R})$ & $\mathfrak{g}_{2(2)}$ & $\mathbf{4}$ & 2 \\
\hline $\mathbb{R} \oplus \mathbb{R}$ & $\mathfrak{s l}(2, \mathbb{R}) \oplus \mathfrak{s l}(2, \mathbb{R})$ & $\mathfrak{s o}(3,4)$ & $(\mathbf{2}, \mathbf{3})$ & 2 \\
\hline $\mathbb{R} \oplus \mathbb{R} \oplus \mathbb{R}$ & $\mathfrak{s l}(2, \mathbb{R}) \oplus \mathfrak{s l}(2, \mathbb{R}) \oplus \mathfrak{s l}(2, \mathbb{R})$ & $\mathfrak{s o}(4,4)$ & $(\mathbf{2}, \mathbf{2}, \mathbf{2})$ & 2 \\
\hline $\mathbb{R} \oplus \boldsymbol{\Gamma}_{m, n}$ & $\mathfrak{s l}(2, \mathbb{R}) \oplus \mathfrak{s o}(m+1, n+1)$ & $\mathfrak{s o}(m+2, n+2)$ & $(\mathbf{2}, \mathbf{m}+\mathbf{n}+\mathbf{2})$ & $2(m=1)$ \\
\hline$J_{3}^{\mathbb{R}}$ & $\mathfrak{s p}(6, \mathbb{R})$ & $\mathfrak{f}_{4(4)}$ & $\mathbf{1 4}^{\prime}$ & 2 \\
\hline$J_{3}^{\mathbb{C}}$ & $\mathfrak{s u}(3,3)$ & $\mathfrak{e}_{6(2)}$ & $\mathbf{2 0}^{\prime}$ & 2 \\
\hline$J_{3}^{\mathbb{C}_{s}}$ & $\mathfrak{s l}(6, \mathbb{R})$ & $\mathfrak{e}_{6(6)}$ & $\mathbf{2 0}$ & 0 \\
\hline$M_{1,2}(\mathbb{O})$ & $\mathfrak{s u}(1,5)$ & $\mathfrak{e}_{6(-14)}$ & $\mathbf{2 0}$ & 5 \\
\hline$J_{3}^{\mathbb{H}}$ & $\mathfrak{s o}{ }^{*}(12)$ & $\mathfrak{e}_{7(-5)}$ & $\mathbf{3 2}^{(\prime)}$ & 2,6 \\
\hline$J_{3}^{\mathbb{H}} \mathbb{H}_{s}$ & $\mathfrak{s o}(6,6)$ & $\mathfrak{e}_{7(7)}$ & $\mathbf{3 2}{ }^{(\prime)}$ & 0 \\
\hline$J_{3}^{\mathbb{Q}}$ & $\mathfrak{e}_{7(-25)}$ & $\mathfrak{e}_{8(-24)}$ & $\mathbf{5 6}$ & 2 \\
\hline$J_{3}^{\mathbb{Q}_{s}}$ & $\mathfrak{e}_{7(7)}$ & $\mathfrak{e}_{8(8)}$ & $\mathbf{5 6}$ & 8 \\
\hline
\end{tabular}

Table 1. Conformal conf $(\widehat{\mathfrak{J}})$ and quasi-conformal qconf $(\widehat{\mathfrak{J}})$ Lie algebras associated to rank-3 Euclidean Jordan algebras. The relevant symplectic irrep. $\mathbf{R}$ of $\mathfrak{c o n f}(\widehat{\mathfrak{J}})$ is also reported. In particular, $\mathbf{1 4}^{\prime}$ denotes the rank-3 antisymmetric irrep. of $\mathfrak{s p}(6, \mathbb{R})$, whereas $\mathbf{3 2}$ and $\mathbf{3 2}^{\prime}$ are the two chiral spinor irreps. of $\mathfrak{s o}^{*}(12)$. Note that $\operatorname{conf}\left(J_{3}^{\mathfrak{L}_{s}}\right)$ and $\mathfrak{q} \mathfrak{c o n f}\left(J_{3}^{\mathfrak{\mathcal { A } _ { s }}}\right)$ are the maximally non-compact (split) real forms of the corresponding compact Lie algebra. $M_{1,2}(\mathbb{O})$ is the JTS generated by $2 \times 1$ vectors over $\mathbb{O}[14,15]$. Note the Jordan algebraic isomorphisms $\boldsymbol{\Gamma}_{1,1} \sim \mathbb{R} \oplus \mathbb{R}$, and $\boldsymbol{\Gamma}_{1,0} \sim \mathbb{R}$. The number of spinor supercharges $\mathcal{N}$ of the corresponding supergravity theory in $D=4$ (cfr. subsection 3.5) is also listed.

potentials sit, along with their duals. As mentioned above, $\operatorname{conf}(\widehat{\mathfrak{J}})$ is nothing but $\operatorname{Inv}\left(\mathfrak{M}_{\widehat{\mathfrak{J}}}\right)$, possibly specified as a suitable non-compact real algebra. ${ }^{18}$

At least in $D=3,4,5,6$, the theories of this class all exhibit (Abelian vector multiplets') scalar manifolds which are symmetric cosets. ${ }^{19}$ In particular, the coset Lie generators in $D=4$ and $D=3$ Lorentzian space-time dimensions are respectively given by $\operatorname{conf}(\widehat{\mathfrak{J}})$ and $\mathfrak{q c o n f}(\widehat{\mathfrak{J}})$ modded out by their maximal compact subalgebra $(\mathrm{mcs})$.

The number of spinor supercharges $\mathcal{N}$ of the $D=4$ supergravity theory is reported in table 1. In particular, the theories associated to $\widehat{\mathfrak{J}}=J_{3}^{\mathfrak{A}} \equiv H_{3}(\mathfrak{A})$ are usually dubbed

\footnotetext{
${ }^{18}$ In fact, as a maximal subalgebra of $\mathfrak{q c o n} \mathfrak{f}(\widehat{\mathfrak{J}})$, in this framework the Lie algebra $\operatorname{Inv}\left(\mathfrak{M}_{\widehat{\mathfrak{J}}}\right)$ can be compact (with commuting subalgebra $\mathfrak{s u}(2)$ ) or non-compact (with commuting subalgebra $\mathfrak{s l}(2, \mathbb{R})$ ), depending on whether the Kaluza-Klein reduction from $D=4 \rightarrow 3$ is performed along a space-like or time-like direction, respectively; in turn, this mathematically corresponds to perform a $c$-map [81] or a $c^{*}$-map (see e.g. [82]) on the $D=4$ (vector multiplets') scalar manifold.

${ }^{19} \mathrm{~A}$ particular case is given by $M_{1,2}(\mathbb{O})$, which (cfr. caption of table 1 ) is a JTS generated by $2 \times 1$ vectors over $\mathbb{O}[14,15]$. It is related to supergravity with 20 local supersymmetries, which exists only in $D=4(\mathcal{N}=5[56])$ and in $D=3(\mathcal{N}=10$; see e.g. [57] and refs. therein).
} 
"magical" MESGT's [14, 15], whereas the $\mathcal{N}=2, D=4$ theories corresponding to $\widehat{\mathfrak{J}}=\mathbb{R}$, $\mathbb{R} \oplus \mathbb{R}$ and $\mathbb{R} \oplus \mathbb{R} \oplus \mathbb{R}$ are the so-called $T^{3}, S T^{2}$ and $S T U$ models [58, 59]. It should also be remarked that $\widehat{\mathfrak{J}}=J_{3}^{\mathbb{H}}$ is related to both $\mathcal{N}=2$ and $\mathcal{N}=6$ theories, which in fact share the very same bosonic sector $[14,15,60-62]$.

As discussed in subsection 2.1, FTS's $\mathfrak{K}(\widehat{\mathfrak{J}})$ (with $\widehat{\mathfrak{J}}$ simple) exhibit a close relationships with exceptional Lie algebras, as given by (2.3). As listed in table 1, when considering suitable non-compact, real forms, (2.3) enjoys the reinterpretation (3.36): in other words, exceptional Lie algebras occur as quasi-conformal Lie algebras of the corresponding simple Jordan algebras $\widehat{\mathfrak{J}}[38,43]$. In this respect, it is worth adding that classical (namely, pseudoothogonal) Lie algebras also occur as quasi-conformal Lie algebras of rank-3 semi-simple Euclidean Jordan algebras of the type ${ }^{20}$ (3.39) [18].

These facts provide indication of possible links between FGT and Yang-Mills (exceptional) gauge theories.

At bosonic level, differences and similarities between the $F G T$ and the class of MESGT's under consideration can be observed by comparing e.g. the $D=3 F G T$ Lagrangian density (3.35) with the bosonic sector of the (ungauged) MESGT $(D=4)$ Lagrangian density (cfr. e.g. the treatment in [63], and refs. therein)

$$
e^{-1} \mathcal{L}=-\frac{1}{2} R-g_{i j} \partial_{\mu} \phi^{i} \partial^{\mu} \phi^{j}+\frac{1}{4} \operatorname{Im}\left(\mathcal{N}_{\Lambda \Sigma}\right) F_{\mu \nu}^{\Lambda} F^{\Sigma \mid \mu \nu}-\frac{e^{-1}}{8} \epsilon^{\mu \nu \rho \sigma} \operatorname{Re}\left(\mathcal{N}_{\Lambda \Sigma}\right) F_{\mu \nu}^{\Lambda} F_{\rho \sigma}^{\Sigma}
$$

Besides the presence of the Einstein-Hilbert term, there are crucial differences: in the $F G T$ the scalar fields $\phi$ fit into $\mathbf{R}(\mathfrak{g})$ and the vectors arise from the gauging of the FTS triple product symmetry algebra $\mathfrak{g}$; as a consequence, the derivatives acting on $\phi$ are covariantized, as discussed in sections 3.2 and 3.3. On the other hand, in the corresponding $(D=4)$ supergravity framework, the Abelian two-form field strengths fit into $\mathbf{R}(\mathfrak{g}=\operatorname{con} \mathfrak{f}(\widehat{\mathfrak{J}}))$, while the scalar fields are in a suitable representation of the maximal compact subalgebra $m c s(\mathfrak{g})$. Furthermore, as discussed above, in $F G T$ the gauge algebra $\mathfrak{g}=\mathfrak{c o n f}(\widehat{\mathfrak{J}})$ and the corresponding global Freudenthal duality are off-shell symmetries of the theory, whereas in the MESGT's under consideration $\mathfrak{g}=\mathfrak{c o n f}(\widehat{\mathfrak{J}})$ is only an on-shell symmetry. ${ }^{21}$ It is also worth pointing out that on the gravity side supersymmetry seems to be an accidental feature; indeed, we recall that for $\widehat{\mathfrak{J}}=J_{3}^{\mathbb{C}_{s}}$ and $J_{3}^{\mathbb{H}_{s}}$, the corresponding theories of gravity coupled to Maxwell and scalar fields are not supersymmetric; possible supersymmetrization of $F G T$ will be discussed in section 4 .

It will be interesting to investigate these relations in future studies; see also the discussion in section 5 .

\footnotetext{
${ }^{20}$ The quasi-conformal realizations constructed in [16-18] correspond to non-linear geometric constructions that leave invariant a generalized lightcone with respect to a quartic distance function. As such, they are different from the algebraic constructions of Lie algebras over triple systems given in the mathematics literature (see e.g. [38, 43]).

${ }^{21}$ One can construct manifestly $U$-invariant Lagrangians, but at the price of a non-manifest Lorentzinvariance [64-66] or of doubling the field strengths' degrees of freedom (doubled formalism [67]; for recent advances in relation to Freudenthal duality, see e.g. [68]).
} 


\section{Generalization?}

In the previous section, we have constructed a consistent Lagrangian for the Freudenthal gauge theory (FGT), based on the FTS $\mathfrak{K}(\mathfrak{J})$, with $\mathfrak{K}$-valued scalar field $\phi(x)$, admitting both (off-shell) FTS gauge symmetry and (off-shell) global Freudenthal-duality symmetry $\mathcal{F}$.

The most important kind of generalization would concern an FGT-type Lagrangian involving some vector fields and/or spinor fields, which is again invariant under both FTS gauge and Freudenthal duality symmetries; indeed, this would be a necessary condition for a supersymmetric (non-trivial) extension of FGT. Moreover, such a generalization is of interest to the physicists, since it potentially might define a sigma-model type theory if the space-time considered in this paper is regarded as the world-volume of some extended objects (for instance, M2-branes), and correspondingly the vector fields conceived as the image of the world-volume in some target space.

However, in subsections 4.1-4.2 we shall prove that, within some minimal reasonable assumptions, such a generalization is not possible.

\subsection{Coupling to a vector space}

Let us start the analysis by coupling a generic $F T S \mathfrak{K}$ to a generic vector space $\mathfrak{V}$, over which one can introduce suitable algebraic structures and make it into an algebra; for instance, spinors can be regarded as vectors with an anti-symmetric binary product that yields the Fermi statistics. In this way, our discussion for the formal algebraic system $\mathfrak{V}$ will cover the most generic space that couples to $\mathfrak{K}$.

Thus, we are considering an extended vector space

$$
\mathfrak{N} \equiv \mathfrak{K} \otimes \mathfrak{V}
$$

whose element, denoted by $\Phi$, is the tensor product of an element $\phi \in \mathfrak{K}$ and an element $v \in \mathfrak{V}$, i.e.

$$
\Phi \equiv \phi \otimes v \in \mathfrak{N}
$$

In order to be able to construct a Lagrangian density functional $\mathbf{L}[\Phi(x)]$ for the fields $\Phi(x) \in \mathfrak{N}$ obtained from promoting an element $\Phi \in \mathfrak{N}$ to a $\mathfrak{N}$-valued space-time field $\Phi(x)$, one starts by introducing a bilinear form (namely, the metric)

$$
\langle\cdot, \cdot\rangle:\left\{\begin{array}{l}
\mathfrak{N} \otimes \mathfrak{N} \rightarrow \mathbb{R} ; \\
\Phi_{I}, \Phi_{J} \mapsto\left\langle\Phi_{I}, \Phi_{J}\right\rangle,
\end{array}\right.
$$

defined for any two $\Phi_{I, J}=\phi_{I, J} \otimes v_{I, J}$ in $\mathfrak{N}$. Via direct evaluation, (4.3) induces a metric on $\mathfrak{V}$ itself:

$$
\left\langle\Phi_{I}, \Phi_{J}\right\rangle=\left\langle\phi_{I} \otimes v_{I}, \phi_{J} \otimes v_{J}\right\rangle=\left\langle\phi_{I}, \phi_{J}\right\rangle \times\left(v_{I}, v_{J}\right)_{\mathfrak{V}}, \quad \forall \Phi_{I}, \Phi_{J} \in \mathfrak{N},
$$


where " $\times$ " is here multiplication by a scalar (real) factor, and

$$
(\cdot, \cdot)_{\mathfrak{V}}:\left\{\begin{array}{l}
\mathfrak{V} \otimes \mathfrak{V} \rightarrow \mathbb{R} \\
v_{I}, v_{J} \mapsto\left(v_{I}, v_{J}\right)_{\mathfrak{V}},
\end{array}\right.
$$

is the induced metric over $\mathfrak{V}$. Note that the symmetry property of $(\cdot, \cdot)_{\mathfrak{V}}(4.5)$ is to be determined by the required symmetry property of the metric $\langle\cdot, \cdot\rangle(4.3)$ over $\mathfrak{N}$ (by also recalling the anti-symmetry of the symplectic form (2.12) over $\mathfrak{K}$ ).

Furthermore, in order to consistently define the Freudenthal duality $\mathcal{F}$ of this extended theory, one needs to introduce a triple product

$$
\mathcal{T}:\left\{\begin{array}{l}
\mathfrak{N} \otimes \mathfrak{N} \otimes \mathfrak{N} \rightarrow \mathfrak{N} \\
\Phi_{I}, \Phi_{J}, \Phi_{K} \mapsto \mathcal{T}\left(\Phi_{I}, \Phi_{J}, \Phi_{K}\right) \equiv \Phi_{I} \Phi_{J} \Phi_{K}
\end{array}\right.
$$

defined for any three elements $\Phi_{I}, \Phi_{J}, \Phi_{K} \in \mathfrak{N}$, which would then induce a tri-linear triple product on $\mathfrak{V}$ itself:

$$
[\cdot, \cdot, \cdot]_{\mathfrak{V}}:\left\{\begin{array}{l}
\mathfrak{V} \otimes \mathfrak{V} \otimes \mathfrak{V} \rightarrow \mathfrak{V} ; \\
v_{I}, v_{J}, v_{K} \mapsto\left[v_{I}, v_{J}, v_{K}\right]_{\mathfrak{V}}
\end{array}\right.
$$

In order to proceed further, we make here a plausible conjecture that Freudenthal duality $\mathcal{F}$ can be defined only for algebraic systems satisfying the axioms $(i)-(i v)$ of an FTS, introduced in subsection 2.3. As a consequence, we require the metric (4.3) to be an anti-symmetric bilinear form (and append this as axiom $(o)$ ), thus obtaining the following five axioms for the algebra $\mathfrak{N}$ :

(o) $\left\langle\Phi_{I}, \Phi_{J}\right\rangle=-\left\langle\Phi_{J}, \Phi_{I}\right\rangle$

(i) $\Phi_{I} \Phi_{J} \Phi_{K}=\Phi_{J} \Phi_{I} \Phi_{K}$

(ii) $\Phi_{I} \Phi_{J} \Phi_{K}=\Phi_{I} \Phi_{K} \Phi_{J}+2 \mu\left\langle\Phi_{J}, \Phi_{K}\right\rangle \Phi_{I}+\mu\left\langle\Phi_{I}, \Phi_{K}\right\rangle \Phi_{J}-\mu\left\langle\Phi_{I}, \Phi_{J}\right\rangle \Phi_{K}$

(iii) $\Phi_{L} \Phi_{M}\left(\Phi_{I} \Phi_{J} \Phi_{K}\right)=\left(\Phi_{L} \Phi_{M} \Phi_{I}\right) \Phi_{J} \Phi_{K}+\Phi_{I}\left(\Phi_{L} \Phi_{M} \Phi_{J}\right) \Phi_{K}+\Phi_{I} \Phi_{J}\left(\Phi_{L} \Phi_{M} \Phi_{K}\right)$

(iv) $\left\langle\Phi_{L} \Phi_{M} \Phi_{I}, \Phi_{J}\right\rangle+\left\langle\Phi_{I}, \Phi_{L} \Phi_{M} \Phi_{J}\right\rangle=0$

where $\mu$ plays the role of the real parameter $\lambda$ introduced above for the FTS $\mathfrak{K}$.

Then, by repeating for the algebra $\mathfrak{N}$ the very same construction discussed in section 3 for the FTS $\mathfrak{K}$, one gets the most general Lagrangian density functional $\mathbf{L}[\Phi(x)]$ invariant under the two desired symmetries, namely under both (off-shell) FTS gauge symmetry and (off-shell) global Freudenthal-duality symmetry $\mathcal{F}$. 


\subsection{A no-go theorem}

However, this seemingly smooth construction of an extended FGT coupled to vector and/or spinor fields suffers from some severe constraints, which actually spoils the above generalization.

Indeed, axioms (o)-(iv) of $\mathfrak{N}$ induce a set of corresponding axioms for the metric (4.5) and the triple product (4.7) induced on $\mathfrak{V}$ (in addition to the ones already introduced for other physical reasons, such as the ones yielded by the Bose and/or Fermi statistics for the fields $\left.v_{I} \in \mathfrak{V}\right)$; the reader can find the full set of such axioms for $\mathfrak{V}$ in appendix C.

Among them, axiom (B. iii) induced from the derivation property of $\mathfrak{N}$ leads to a particularly strong constraint. In order to realize this, let us restrict to a subalgebra

$$
\mathfrak{N}_{\phi} \equiv \mathfrak{K}_{\phi} \otimes \mathfrak{V} \subset \mathfrak{N}
$$

where $\mathfrak{K}_{\phi}$ is the subalgebra in $\mathfrak{K}$ generated by a single generator $\phi \in \mathfrak{K}$ (see also subsection 3.1). Then, by taking five elements of the form

$$
\Phi_{L, M, I, J, K} \equiv \phi \otimes v_{L, M, I, J, K} \in \mathfrak{N}_{\phi}
$$

and inserting them into axiom (B.iii) of appendix C, the following simplified (weaker) condition on the algebraic structure of $\mathfrak{V}$ is achieved:

$$
\phi \phi T(\phi) \otimes\left(\left[v_{L}, v_{M},\left[v_{I}, v_{J}, v_{K}\right]_{\mathfrak{V}}\right]_{\mathfrak{V}}-\left[v_{I}, v_{J},\left[v_{L}, v_{M}, v_{K}\right]_{\mathfrak{V}}\right]_{\mathfrak{V}}\right)=0,
$$

where the simplification comes from the fact that over the subalgebra $\mathfrak{K}_{\phi}, \mathcal{L}_{\phi T(\phi)}$ and $\mathcal{L}_{T(\phi) \phi}$ act as annihilation operators, whose proof can be found in appendix A.

Moreover, we observe that, as holding for $\mathfrak{K}$ (cfr. definition (2.13)) for any two elements $v_{L}, v_{M} \in \mathfrak{V}$ one gets a linear operator (generally $\mathfrak{g l}(\mathfrak{V})$-valued, whenever it is non-zero) $\mathcal{L}_{v_{L} v_{M}}$, whose action is evaluated by the triple product (4.7) as:

$$
\mathcal{L}_{v_{L} v_{M}}:\left\{\begin{array}{l}
\mathfrak{V} \otimes \mathfrak{V} \rightarrow \mathfrak{V} ; \\
v_{L}, v_{M} \mapsto \mathcal{L}_{v_{L} v_{M}} v_{I} \equiv\left[v_{L}, v_{M}, v_{I}\right]_{\mathfrak{V}},
\end{array}\right.
$$

Then, by using definition (4.11), the weaker form (4.10) of the axiom (B.iii) can be recast as a condition on the matrix commutator in $\mathfrak{g l}(\mathfrak{V})$ :

$$
\left[\mathcal{L}_{v_{L} v_{M}}, \mathcal{L}_{v_{I} v_{J}}\right]=0, \forall v_{I, J, L, M} \in \mathfrak{V} .
$$

Under the assumption that the metric (4.3) in $\mathfrak{N}$ is non-degenerate (which we understand throughout ${ }^{22}$ ), the condition (4.12) can be satisfied in only two instances:

\footnotetext{
${ }^{22}$ For instance, if the metric (4.3) of the algebra $\mathfrak{N}$ is degenerate, it can be proved that a coupling to a Grassmannian number degree of freedom is possible. However, since there is no fermion bilinear for a one-dimensional spinor, this is a rather trivial theory, in which the fermionic degree of freedom never plays any role, and it cannot mix up with the bosonic degree of freedom. In such a theory, the structure is essentially the same as the one pertaining to a single $\mathfrak{K}$-valued (real) scalar field, and hence a consistent implementation of invariance under (global, off-shell) Freudenthal duality is possible. We plan to investigate further this issue in future work.
} 
[I] when $\operatorname{dim}_{(\mathbb{R})} \mathfrak{V}=1$, i.e.

$$
\mathfrak{N}=\mathfrak{K} \otimes \mathbb{R},
$$

which is the case of a single $\mathfrak{K}$-valued (real) scalar field discussed in sections $2-3$;

[ III ] when the set

$$
\left\{\mathcal{L}_{v_{I} v_{J}} \in \mathfrak{g l}(\mathfrak{V}) \mid v_{I}, v_{J} \in \mathfrak{V}\right\} \subset \mathfrak{g l}(\mathfrak{V})
$$

is a subset of the Cartan subalgebra of $\mathfrak{g l}(\mathfrak{V})$, namely ${ }^{23}$ (recall definitions (4.5) and (4.11)):

$$
\mathcal{L}_{v_{I} v_{J}} v_{K}=\left[v_{I}, v_{J}, v_{K}\right]_{\mathfrak{V}}=\left(v_{I}, v_{J}\right)_{\mathfrak{V}} \times v_{K} .
$$

The triple product $[\cdot, \cdot, \cdot]_{\mathfrak{V}}(4.7)$ defined by $(4.15)$ satisfies the strong form of axiom (B. iii) and most of other axioms of appendix C. However, at least within the assumption of non-degeneracy of the metric of the algebra $\mathfrak{N}$ (cfr. footnote 19), it is refuted by axiom $(B . i i)$ whenever $\mathfrak{K}$ is larger then a single-generator algebra $\mathfrak{K}_{\phi}$.

This completes the proof of the following

No-go theorem. Assuming the metric of the algebraic system $\mathfrak{N}$ (4.1) to be nondegenerate and the Freudenthal duality $\mathcal{F}$ to be defined only for $\mathfrak{N}$ satisfying all the four FTS axioms introduced in subsection 2.3, then it is not possible to construct a Lagrangian density functional $\mathbf{L}[\Phi(x)]$ for a $\mathfrak{K}$-valued vector/spinor field $\Phi(x)$ which admits both (off-shell) FTS gauge symmetry and (off-shell) global F-duality symmetry $\mathcal{F}$.

\section{$5 \quad$ FGT and $(\mathcal{N}=3, D=3)$ SC CSM gauge theories}

We will now briefly make some observations on the relation between Freudenthal gauge theory (FGT) (based on Freudenthal triple systems (FTS's)) and the intense research on triple systems and gauge theories, in which remarkable advances were achieved after the seminal papers of Bagger and Lambert [10] and Gustavsson [11]. A more detailed analysis will be reported in [34].

Here, we will focus on the relation to superconformal (SC) Chern-Simons-matter (CSM) gauge theories in $D=3$ (in which the $\mathcal{R}$-symmetry structure is richer); we will mainly refer to the mathematical treatment of [36] and [37] (see also [69]); for an extensive list of refs. on BLG theories and related developments, besides [36, 37, 69], we address the reader e.g. to the recent comprehensive review [12].

We anticipate that the symmetry properties (2.20) of the FTS structure constants on which the FGT is based are generally different from the ones pertaining to the structure

\footnotetext{
${ }^{23}$ In general, instead of (4.15) one may propose

$$
\mathcal{L}_{v_{I} v_{J}} v_{K}=\left[v_{I}, v_{J}, v_{K}\right]_{\mathfrak{V}}=h\left(\left(v_{I}, v_{J}\right)_{\mathfrak{V}}\right) \times v_{K},
$$

for any function $h: \mathbb{R} \rightarrow \mathbb{R}$, as the most generic possibility [ $\mathbb{I I}]$. However, the tri-linearity of the triple product $[\cdot, \cdot, \cdot]_{\mathfrak{V}}(4.7)$ in $\mathfrak{V}$ requires the function $h$ to be linear. Since the constant term of the linear function $h$ leads to a trivial triple product and is easily refuted by the other axioms of appendix $\mathrm{C}$, one can conclude that, up to an overall (real) factor, (4.15) is the most generic possibility [ III ].
} 
constants on which the BLG-type theories (such as the ones investigated e.g. in [70, 71], among others) rely. Among SC CSM $D=3$ gauge theories, the symmetry (2.20) is indeed consistent only with $\mathcal{N}=3$ (see e.g. [37], and refs. therein). Disregarding the global (off-shell) Freudenthal duality, $(D=3)$ FGT could be viewed as an alternative, purely bosonic sector of the corresponding $\mathcal{N}=3, D=3$ SC CSM gauge theory. In fact, as analyzed in section 3.3, in FGT the non-vanishing of $f_{(a b c d)}$ allows for terms in the Lagrangian which differ from the usual ones in BLG theories; for instance, the simplest FGT scalar potential is quartic in the scalar fields (essentially given by $\Delta(2.21)$; see (3.29)), whereas in BLG theories it is of order six (see e.g. (19) of [10]).

We start by observing that the set of axioms (i), (iii) and (iv) defining an FTS (as given in section 2.4) match the set of axioms $(a),(b)$ and $(c)$ defining the triple systems based on quaternionic unitary representations $\mathbf{W}$ of a metric Lie algebra $\mathfrak{g}$, as discussed in [36] and [37] (see e.g. appendix A.2.4 of [37], and axioms (125)-(127) therein); in particular, the FTS axiom (iii) is nothing but the so-called fundamental identity of the triple system (see e.g. (127) of [37]). In turn, the treatment of [36] and [37] is based on a construction due to Faulkner [35, 72], which essentially constructs triple systems from pairs $(\mathfrak{g}, \mathbf{V})$, where $\mathbf{V}$ is a suitable representation ${ }^{24}$ of $\mathfrak{g}[36]$.

The $\mathfrak{g}$-irreducible decomposition of the rank- $4 \mathfrak{g}$-invariant structure in $\mathbf{W}$ is given by (124) of [37] (also, cfr. table 2 therein):

$$
S^{2} S^{2} \mathbf{W} \cong S^{4} \mathbf{W} \oplus \mathbf{W}^{(2,2)}
$$

In tensor notation, a reformulation ${ }^{25}$ of (5.1) reads as follows $(a, b \in \mathbb{R})$ :

$$
f_{a b c d}=a f_{(a b c d)}+b \omega_{a(c} \omega_{d) b} .
$$

(5.2) is consistent with the general symmetry of the FTS structure constants' tensor $f_{a b c d}$ given by (2.20); furthermore, Freudenthal duality $\mathcal{F}(2.25)$ can be consistently introduced whenever $f_{(a b c d)} \neq 0$.

It is worth remarking that Brown's definition of Lie algebra $(\mathfrak{g}, \mathbf{R})$ of type $\mathfrak{e}_{7}[22]$ (cfr. $(a)-(c)$ in section 2.5) can be extended to include also the not completely symmetric part $\omega_{a(c} \omega_{d) b}$ of (5.2) as follows: $\mathbf{R}$ is a representation space of $\mathfrak{g}$ such that

( $\widehat{a}) \mathbf{R}$ possesses a non-degenerate, skew-symmetric bilinear $\mathfrak{g}$-invariant form $\omega$ (cfr. (2.12) and $(2.17))$;

$(\widehat{b}) \mathbf{R}$ possesses a rank-4 $\mathfrak{g}$-invariant structure $f_{a b c d}(5.2)$, which allows to define

$$
\widehat{q}(x, y, z, w) \equiv f_{a b c d} x^{a} y^{b} z^{c} w^{d}=2 \widehat{\Delta}(x, y, z, w) ;
$$

$(\widehat{c})$ by defining a ternary product $\widehat{\mathbf{T}}(x, y, z)$ on $\mathbf{R}$ as

$$
\langle\widehat{\mathbf{T}}(x, y, z), w\rangle \equiv \widehat{q}(x, y, z, w),
$$

\footnotetext{
${ }^{24}$ The fourth axiom (quaternionic condition; see e.g. (128) of [37]) defining Faulkner's triple systems based on $(\mathfrak{g}, \mathbf{W})$ is essentially related to the existence of a skew-symmetric symplectic invariant bilinear form $\omega$ which raises and lowers indices.

${ }^{25}$ Here, we will not deal with issues of generality of the reformulation (5.2) of (5.1).
} 
then one has

$$
3\langle\widehat{\mathbf{T}}(x, x, y), \widehat{\mathbf{T}}(y, y, y)\rangle=\langle x, y\rangle \widehat{q}(x, y, y, y) .
$$

By enhancing $f_{a b c d}=f_{(a b c d)}$ to a not completely symmetric $f_{a b c d}$ given by (5.2), one can conclude that, by virtue of $(\widehat{a})$, the real parameters $a$ and $b$ can always be chosen such that the inclusion of $\omega_{a(c} \omega_{d) b}$ in Brown's definition [22] yields nothing but an equivalent definition of a Lie algebra of type $\mathfrak{e}_{7}$; however, as pointed out below, the presence or absence of the term $\omega_{a(c} \omega_{d) b}$ matters in order to make contact with FTS's.

Note that the $\lambda$-dependent FTS-defining axiom (ii) was not mentioned so far. However, at least for the class of pairs $(\mathfrak{g}, \mathbf{R})=(\mathfrak{c o n f}(\widehat{\mathfrak{J}}), \mathbf{R})$ reported in table 1 , the parameters $a$ and $b$ can be fixed consistently with axiom (ii), by further elaborating (5.2) as

$$
f_{a b c d}=6 \lambda f_{(a b c d)}-2 \lambda \omega_{a(c} \omega_{d) b} .
$$

For pairs $(\mathfrak{g}, \mathbf{R})=(\mathfrak{c o n f}(\widehat{\mathfrak{J}}), \mathbf{R})$ with $\mathfrak{g}$ simple, both (5.6) and the parameter $\lambda$ acquires a very simple group-theoretical meaning. Indeed, exploiting the results of [73], (5.6) can be rewritten as

$$
f_{a b c d}=-3 \tau f_{(a b c d)}+\tau \omega_{a(c} \omega_{d) b}=t_{a b}^{\alpha} t_{c d}^{\beta} g_{\alpha \beta},
$$

where $t_{a b}^{\alpha}=t_{(a b)}^{\alpha}$ is the ( $\mathfrak{g}$-invariant) realization of the generators of $\mathfrak{g}$ in $\mathbf{R}$; the indices $\alpha$ and $a$ respectively are in Adj and $\mathbf{R}$ of $\mathfrak{g}$, whose Cartan-Killing metric is $g_{\alpha \beta}$. Therefore, $f_{a b c d}$ can be defined as the adjoint-trace of the product of two realizations of generators of $\mathfrak{g}$ in its representation $\mathbf{R}$. Moreover, the parameter [73]

$$
\tau \equiv \frac{2 \operatorname{dim}_{\mathbb{R}} \mathbf{A d j}(\mathfrak{g})}{\operatorname{dim}_{\mathbb{R}} \mathbf{R}(\mathfrak{g})\left(\operatorname{dim}_{\mathbb{R}} \mathbf{R}(\mathfrak{g})+1\right)}=-2 \lambda
$$

expresses the ratio between the sets of indices $\alpha$ and $a b=(a b)$ of $t_{a b}^{\alpha}$ (in the treatment above, we set $\operatorname{dim}_{\mathbb{R}} \mathbf{R}(\mathfrak{g}) \equiv f$; cfr. (2.6)). By virtue of the Gaillard-Zumino embedding (2.5) [44] (or, equivalently of the aforementioned Theorem by Dynkin $[45,46]$ ), $\tau$ expresses the fraction of generators of $\mathfrak{s p}(f, \mathbb{R})$ which generate its maximal (generally non-symmetric) sub-algebra $\mathfrak{g}$. Indeed, it holds that

$$
0<\tau \leqslant 1 \Leftrightarrow-\frac{1}{2} \leqslant \lambda<0 .
$$

By a suitable generalization of the analysis of [83], explicitly worked out in [71], the choice of $f_{a b c d}$ given by $(5.7)$ can be made also for the pairs $(\mathfrak{g}, \mathbf{R})=(\mathfrak{c o n f}(\widehat{\mathfrak{J}}), \mathbf{R})$ with $\mathfrak{g}$ semi-simple. However, in these cases the last step of (5.2) does not hold:

$$
f_{a b c d}=-3 \tau f_{(a b c d)}+\tau \omega_{a(c} \omega_{d) b} \neq t_{a b}^{\alpha} t_{c d}^{\beta} g_{\alpha \beta}
$$

in fact, the explicit expression of $t_{a b}^{\alpha} t_{\alpha \mid c d}$ for these cases has been computed in [71], and it is such that [70]

$$
g_{\alpha \beta} t_{(a b}^{\alpha} t_{c) d}^{\beta}=0
$$


Thus, the FTS (the triple system on which the FGT is based) turns out to be related to the quaternionic level of Faulkner's construction [35] of triple systems from pairs $(\mathfrak{g}, \mathbf{V})$, which has been recently re-analyzed by $[36,37,69]$ within $D=3$ SC CSM gauge theories.

An important difference with the latter framework is the fact that, in the treatment of the present paper, FTS is defined on the ground field $\mathbb{R}$ (recall footnote 1); this constrains the pair $(\mathfrak{g}, \mathbf{V})=(\mathfrak{g}, \mathfrak{K})$ such that $\mathbf{V}$ is a real representation space of the (non-compact) real algebra $\mathfrak{g}$; some examples, related to conformal symmetries of JTS $\mathfrak{J}=\widehat{\mathfrak{J}}$, are reported in table 1. As mentioned in section 3.3, we point out that this is not inconsistent with the physical constraint on matter representations in $D=3$ SC CSM gauge theories; indeed, $\mathbf{V}=\mathbf{W}$ is always assumed to possess a positive-definite inner product (for unitarity of the corresponding gauge theory), but CS gauge fields are not propagating (and they are in $\operatorname{Adj}(\mathfrak{g})$ ), and therefore $\mathfrak{g}$ does not necessarily have to be endowed with a positive-definite product, thus allowing for non-compact (real) forms of $\mathfrak{g}$.

The expression (5.2) of the FTS structure constants' tensor $f_{a b c d}$ (or, equivalently, for the rank-4 $\mathfrak{g}$-invariant structure in $\mathbf{W}$ in $(\mathfrak{g}, \mathbf{V}=\mathbf{W})$-based Faulkner's construction of triple systems [35]) entails two "extremal" cases:

1. The case in which $f_{a b c d}$ is completely symmetric (and therefore Freudenthal duality $\mathcal{F}(2.25)$ can be consistently introduced). This corresponds to $b=0$ and (up to redefinition) $a=1$ in (5.2):

$$
f_{a b c d}=f_{(a b c d)},
$$

which characterizes Brown's definition [22] of $(\mathfrak{g}, \mathbf{W})$ as a Lie algebra of type $\mathfrak{e}_{7}$ (cfr. axiom (b) in section 2.5). The corresponding triple system has been called quaternionic Lie triple system ( $q L T S)$ in [37]. However, this triple system is not relevant for application to (BLG-type) gauge theories. Indeed, for positive-definite $\mathbf{W}$ (as assumed for unitarity of the corresponding gauge theory), $f_{a b c d}$ is nothing but the Riemann tensor of a symmetric hyper-Kähler manifold, which is Ricci-flat; however, any homogeneous Ricci-flat Riemannian manifold is actually Riemann-flat [84, 85]. Thus, a positive-definite $\mathbf{W}$ in qLTS (5.11) is necessarily the trivial representation (cfr. corollary 6 in [37]). Remarkably, this result has a consistent interpretation in the FTS framework. Indeed, it can be checked that (5.11), when plugged into the FTS axiom (iii) (fundamental identity) and contracted with $x^{a} x^{b} y^{c} y^{e} y^{f} y^{g}$, does not yield the axiom $(c)$ which defines a Lie algebra of type $\mathfrak{e}_{7}[22]$. In other words, $(\mathfrak{g}, \mathbf{W})$ of type $\mathfrak{e}_{7}[22]$ is not consistent with the FTS introduced in sections 2.5-2.4; in particular, the fundamental identity (iii) is not consistent with axiom (c) of Lie algebras of type $\mathfrak{e}_{7}[22]$. As a consequence, the limit of the defining axioms $(i)-(i v)$ in which $f_{a b c d}$ is taken to be completely symmetric (5.11) is ill defined; a non-trivial $\lambda \rightarrow 0$ limit in $(i)-(i v)$ can still be implemented, but it yields an FTS which does not fulfill the symmetry condition (5.11) [34].

2. The case in which $f_{a b c d}$ lacks its completely symmetric part. This corresponds to $a=0$ and (up to redefinition) $b=1$ in (5.2):

$$
f_{a b c d}=\omega_{a(c} \omega_{d) b}
$$


In this case the Freudenthal duality $\mathcal{F}(2.25)$ cannot be consistently introduced. The corresponding triple system has been called anti-Lie triple system (aLTS) in [37]; it characterizes $\mathcal{N}=4$ and $\mathcal{N}=5$ SC CSM gauge theories in $D=3$, as thoroughly analyzed in [37] (see also table 6 therein), by elaborating on previous literature (see refs. therein). A prototypical case (treated in Example 1 of [41]) is provided by a consistent limit of $(5.6)$, given by $^{26}$ (recall $\left.(2.6)\right) \mathfrak{g}=\mathfrak{s p}(f, \mathbb{R})$ and $\mathbf{W}=\mathbf{f}$ (fundamental irrep.). Since

$$
S^{4} \mathbf{f} \equiv(\mathbf{f} \times \mathbf{f} \times \mathbf{f} \times \mathbf{f})_{s}
$$

is irreducible in $\mathfrak{s p}(f, \mathbb{R})$ and contains no singlets, it follows that $f_{(a b c d)}=0$. On the other hand, since $\operatorname{Adj}(\mathfrak{s p}(f, \mathbb{R}))=S^{2} \mathbf{f} \equiv(\mathbf{f} \times \mathbf{f})_{s}$, the definition (5.8) also yields $\tau=1$, and therefore (5.12) is recovered from (5.6). It is worth remarking that in this case the resulting FTS is not endowed with a manifestly JTS-covariant structure (2.1) as in the original Freudenthal's formulation [38-40]; the corresponding (super)gravity theory in $D=4$ can have at most ${ }^{27} \mathcal{N}=1$ local supersymmetry, and has a (nonspecial) Kähler scalar coset with algebra $\mathfrak{s p}(f, \mathbb{R}) \ominus \mathfrak{u}(f / 2)$ (upper Siegel half-plane).

The general triple system under consideration, which interpolates between $q L T S$ (5.11) and aLTS (5.12), is endowed with an $f_{a b c d}$ given by (5.2) with both $a$ and $b$ non-vanishing. As anticipated, among SC CSM gauge theories in $D=3$, this is consistent only with $\mathcal{N}=3$ (see e.g. [37], and refs. therein), which is thus the only amount of (global) supersymmetry for which Freudenthal duality $\mathcal{F}(2.25)$ could a priori be implemented, even if its enforcement as a global (off-shell) symmetry is in contrast with supersymmetry itself, as implied by the No-Go theorem proved in section 4.2.

It is worth observing that this general case is also consistent with the "extension" of the definition of Lie algebras of type $\mathfrak{e}_{7}$ (based on axioms $(\widehat{a})-(\widehat{c})$ above); indeed, up to some redefinitions, the real parameters $a$ and $b$ can always be chosen such that (5.2), when plugged into the FTS axiom (iii) and contracted with $x^{a} x^{b} y^{c} y^{e} y^{f} y^{g}$, does yield the axiom $(\widehat{c})$ introduced above; the term $\omega_{a(c} \omega_{d) b}$ plays a key role in this result.

The above treatment hints for the existence of a class of $\mathcal{N}=3, D=3$ SC CSM gauge theories in which the gauge Lie algebra and its matter representation are given by

$$
(\mathfrak{g}, \mathbf{V})=(\mathfrak{c o n f}(\widehat{\mathfrak{J}}), \mathbf{R}),
$$

namely they are respectively given by the conformal symmetries of rank-3, Euclidean Jordan algebras, and by their relevant symplectic irreps. $\mathbf{R}$, as reported in table 1 .

In this respect, by recalling section $3.5, \mathcal{N}=3, D=3 \mathrm{SC}$ CSM gauge theories based on (5.14) share the same symmetry (with different physical meanings) of two other distinct classes of theories:

- $D=4$ Maxwell-Einstein (super)gravity theories (ME(S)GT) (with various amount $\mathcal{N}$ of local supersymmetry) having symmetric scalar manifolds, as discussed in section 3.5 (and reported in table 1);

\footnotetext{
${ }^{26}$ Recall that, under the assumption that $\omega$ is non-degenerate, $f$ is even.

${ }^{27}$ In this theory, the consistency of $\mathcal{N}=1$ local supersymmetry with a symplectic structure of electric and magnetic fluxes has been studied e.g. in [53]; see also [86].
} 
- $D=3$ Freudenthal gauge theories (FGT's) based on an FTS $\mathfrak{K} \sim \mathbf{R}(\operatorname{con} \mathfrak{f}(\widehat{\mathfrak{J}}))$. The consistency of FGT with (global) supersymmetry is an important difference with respect to $\mathcal{N}=3 \mathrm{SC}$ CSM gauge theories. Indeed, the No-Go Theorem proved in section 4.2 essentially states that global (off-shell) Freudenthal duality is not consistent with a non-trivial coupling to space-time vector/spinor fields, which in turn is a necessary condition for supersymmetry.

These relations among $\mathcal{N}=3, D=3 \mathrm{SC}$ CSM gauge theories, $D=4 \mathrm{ME}(\mathrm{S}) \mathrm{GT}$ 's and FGT's can actually be extended to the general case in which the pair $(\mathfrak{g}, \mathbf{V}=\mathbf{W})$ defines a generic FTS (based on axioms $(i)-(i v)$ ) corresponding, in the sense outlined above, to the "quaternionic level" of Faulkner's construction [35-37, 69, 72].

We plan to investigate this interesting interplay of symmetries in future work [34] (also in view of possible AdS/CFT applications). In particular, as anticipated above, when disregarding the global (off-shell) Freudenthal duality, it would be interesting to consider the consistency of $(D=3)$ FGT as an alternative, purely bosonic sector of the corresponding $\mathcal{N}=3, D=3 \mathrm{SC}$ CSM gauge theory. In fact, as analyzed in section 3.3, in FGT the non-vanishing of $f_{(a b c d)}$ allows for terms in the Lagrangian which differ from the usual ones in BLG theories; for instance, the simplest FGT scalar potential is quartic in the scalar fields (essentially given by $\Delta(2.21)$; see (3.29)), whereas in BLG theories it is of order six (see e.g. (19) of [10]).

\section{Concluding remarks}

In this paper, we have introduced the Freudenthal Gauge Theory (FGT), a gauge theory invariant under two off-shell symmetries: a local, gauge symmetry constructed from a Freudenthal Triple System $(F T S) \mathfrak{K}$, and a global symmetry based on the so-called Freudenthal Duality (F-duality) $\mathcal{F}$.

We have presented the most general bosonic action invariant under these two symmetries, containing a single $\mathfrak{K}$-valued scalar field $\phi(x)$ and a gauge field $A_{\mu}^{a b}(x) \in \mathfrak{K} \otimes_{S} \mathfrak{K}$. The algebraic structure of the FTS ensures that the FGT is well defined and has the required properties.

One of the building blocks of FGT is the $F$-duality $\mathcal{F}$, which is a non-linear anti-involutive duality $\left(\mathcal{F}^{2}=-I d\right)$ which gives, up to a sign, a one-to-one pairing of elements in $\mathfrak{K}$.

In section 4 , we have also analyzed the possibility of generalizing the simple setup presented in section 3 by coupling to space-time vector and/or spinor fields, which is a necessary condition for supersymmetry and is usually a relatively simple step in the construction of gauge theories. Within the assumption ${ }^{28}$ that Freudenthal duality $\mathcal{F}$ can be defined only for algebraic systems satisfying the FTS axioms (i)-(iv) (see subsection 2.3) we have proved a No-Go theorem (which holds true if the metric of the system is nondegenerate), which essentially forbids the coupling to space-time vector and/or spinor fields.

\footnotetext{
${ }^{28}$ We leave the possible relaxation of the assumptions on $\mathcal{F}$ and/or on the metric of the algebraic system to further future investigation. Concerning the case of degenerate metric, see also footnote 19.
} 
However, we point out that such a coupling is possible at least if one relaxes the requirement of invariance under F-duality. Despite the fact that in our treatment there is, a priori, no restriction on the space-time dimension $D$, non-compact gauge Lie algebras g generally yield non-unitary theories in $D \geqslant 4$ (cfr. the remark below (3.33)). However, in $D=3$ this is no more a problem, and the resulting (non-Freudenthal-invariant) FGT can contain both bosonic and fermionic degrees of freedom together with the Chern-Simons term.

In $D=3$, some intriguing similarities (and important differences) between FGT and superconformal (SC) Chern-Simons-matter (CSM) gauge theories with $\mathcal{N}=3$ global supersymmetry have been discussed in section 5. Indeed, among SC CSM gauge theories in $D=3$, a generic FTS is only consistent for $\mathcal{N}=3$ (see e.g. [37], and refs. therein), which is thus the only amount of (global) supersymmetry for which Freudenthal duality $\mathcal{F}(2.25)$ could a priori be implemented, even if its enforcement as a global (off-shell) symmetry is in contrast with supersymmetry itself, as implied by the No-Go theorem proved in section 4.2.

It is worth recalling here that our treatment hints for the existence of a class of $\mathcal{N}=3$, $D=3$ SC CSM gauge theories in which the gauge Lie algebra is given by (5.14), namely by the conformal algebras $\mathfrak{g}=\mathfrak{c o n f}(\widehat{\mathfrak{J}})$ of rank-3, Euclidean Jordan algebras, and by their relevant symplectic irreps. $\mathbf{R}$, as reported in table 1 . In this respect, such $\mathcal{N}=3, D=3$ SC CSM gauge theories share the same symmetry (with different physical meanings) of two other distinct classes of theories: I] $D=4$ Maxwell-Einstein (super)gravity theories $(\mathrm{ME}(\mathrm{S}) \mathrm{GT})$ (with various amount $\mathcal{N}$ of local supersymmetry) with symmetric scalar manifolds, as discussed in section 3.5 (and reported in table 1); II $] D=3$ FGT's based on an $F T S \mathfrak{K} \sim \mathbf{R}(\operatorname{conf}(\widehat{\mathfrak{J}}))$.

These relations among $\mathcal{N}=3, D=3 \mathrm{SC}$ CSM gauge theories, $D=4 \mathrm{ME}(\mathrm{S}) \mathrm{GT}$ 's and $D=3$ FGT's can actually be extended to the general case in which the pair $(\mathfrak{g}, \mathbf{V}=\mathbf{W})$ defines a generic FTS (based on axioms $(i)-(i v)$ ) corresponding, as discussed in section 5, to the "quaternionic level" of Faulkner's construction [35-37, 69, 72].

We plan to investigate this interesting interplay of symmetries in future work [34] (also in view of possible AdS/CFT applications). In particular, when disregarding the global (off-shell) Freudenthal duality, it will be interesting to consider the consistency of $D=3$ FGT as an alternative, purely bosonic sector of the corresponding $\mathcal{N}=3, D=3 \mathrm{SC}$ CSM gauge theory. In fact, as analyzed in section 3.3, in FGT the non-vanishing of $f_{(a b c d)}$ allows for terms in the Lagrangian which differ from the usual ones in BLG theories; for instance, the simplest FGT scalar potential is quartic in the scalar fields (essentially given by $\Delta$ (2.21); see (3.29)), whereas in BLG theories it is of order six (see e.g. (19) of [10]).

The close relation between the particular class $\mathfrak{K}(\widehat{\mathfrak{J}})$ of $F T S$ 's and exceptional Lie algebras $\mathfrak{g}$ (discussed in sections 2.1 and 3.4) could also be used to investigate the possible relation (duality?) between FGT and Yang-Mills gauge theory with exceptional gauge Lie algebra $\mathfrak{g}$. This is certainly possible, but one should recall that exceptional Lie groups cannot be embedded into standard matrix groups, and thus the resulting Yang-Mills theory would not have the standard Maxwell term constructed from trace over matrices. Geometrically, a better way to understand this model is by noting that the exceptional Lie groups can be embedded as matrix groups over octonions $\mathbb{O}[74]$; thus, the $\mathfrak{K}(\widehat{\mathfrak{J}})$-based FGT would be dual to a standard Yang-Mills theory over ${ }^{29} \mathbb{O}$.

\footnotetext{
${ }^{29}$ For similar formulations, see e.g. [75-77], and refs. therein.
} 
The present investigation proved the quartic polynomial $\Delta(2.21)$ to be invariant not only under Freudenthal duality $\mathcal{F}(2.25)$, but also under the (global or gauged) transformation based on the FTS triple product (2.11). It will be interesting to investigate the physical meaning of such an invariance of $\Delta$ e.g. within black hole physics [23] and flux compactifications [79], in which $\Delta$ occurs in relation respectively to the Bekenstein-Hawking [32, 33] black hole entropy and to the cosmological constant. Interesting recent advances on Freudenthal duality $[68,87]$ might also lead to further developments in understanding FGT.

Finally, we would like to point out that the FTS has another intriguing geometrical interpretation in terms of the so-called metasymplectic geometry, introduced decades ago by Freudenthal [38] [88]. In such a geometric framework, two points can define, instead of a line passing through them as in the standard geometry, two more relations, called interwoven and hinged. Furthermore, to each set of points there corresponds a set of dual geometrical objects called symplecta, satisfying relations which are dual to the aforementioned three ones among the points. In this bizarre geometrical setup, the FTS axioms acquire a natural geometrical interpretation, and the relation to the exceptional Lie algebras becomes more transparent. We leave the possible physical interpretation of such a fascinating geometry within FGT for further future investigation.

\section{Acknowledgments}

We are grateful to Raymond Stora for encouragement, enlightening discussions and careful reading of the manuscript.

A.M. would like to thank Rob Knoops for discussions.

A.M. would also like to thank the Department of Physics, University of California at Berkeley, where part of this project was done, for kind hospitality and stimulating environment.

The work of B. Z. has been supported in part by the Director, Office of Science, Office of High Energy and Nuclear Physics, Division of High Energy Physics of the U.S. Department of Energy under Contract No. DE-AC02-05CH11231, and in part by NSF grant 30964-13067-44PHHXM.

\section{A Freudenthal duality}

In this appendix, generalizing the treatment of [23] (in turn referring to [22]) to a generic FTS $\mathfrak{K}$ (see also [24]), we present the proof that the quartic polynomial $\Delta(\phi)(2.21)$ is invariant under the Freudenthal duality $\mathcal{F}(2.25)$.

By recalling definition (2.13), we can restate the derivation property (FTS axiom $\left.\left(i i i^{\prime}\right)\right)$ as follows:

$$
\left[\mathcal{L}_{\phi_{L} \phi_{M}}, \mathcal{L}_{\phi_{I} \phi_{J}}\right] \phi_{K}=\mathcal{L}_{\left(\phi_{L} \phi_{M} \phi_{I}\right) \phi_{J}} \phi_{K}+\mathcal{L}_{\phi_{I}\left(\phi_{L} \phi_{M} \phi_{J}\right)} \phi_{K}
$$

Since this equation is true for any element $\phi_{K} \in \mathfrak{K}$, it is true as an operator equation for $\mathcal{L}_{\phi_{I} \phi_{J}}$. Setting $I=J=L=M$, we find that

$$
\left[\mathcal{L}_{\phi \phi}, \mathcal{L}_{\phi \phi}\right]=\mathcal{L}_{T(\phi) \phi}+\mathcal{L}_{\phi T(\phi)}=2 \mathcal{L}_{\phi T(\phi)}
$$


where the FTS axiom $(i)$ of subsection 2.3 has been used. Since the commutator of an operator with itself must vanish, the above equation must be equal to zero:

$$
\mathcal{L}_{\phi T(\phi)}=0
$$

This means, again by the derivation property of $\mathcal{L}$, that both $\mathcal{L}_{T(\phi) \phi}$ and $\mathcal{L}_{\phi T(\phi)}$ act like annihilation operators on any element $\phi_{K} \in \mathfrak{K}$.

Then, by recalling the definition (2.21), from the FTS axiom (ii) of subsection 2.3 one obtains:

$$
\begin{aligned}
\mathcal{L}_{T(\phi) T(\phi)} \phi & =T(\phi) T(\phi) \phi \\
& =T(\phi) \phi T(\phi)+2 \lambda\langle T(\phi), \phi\rangle T(\phi)+\lambda\langle T(\phi), \phi\rangle T(\phi)-\lambda\langle T(\phi), T(\phi)\rangle \phi \\
& =6 \lambda \Delta(\phi) T(\phi) ; \\
\mathcal{L}_{\phi \phi} T(\phi) & =\phi \phi T(\phi)=-6 \lambda \Delta(\phi) \phi .
\end{aligned}
$$

Consequently, the direct evaluation of $T(T(\phi))$ reads:

$$
\begin{aligned}
T(T(\phi)) & =\mathcal{L}_{T(\phi) T(\phi)} T(\phi)=6 \lambda \Delta(\phi)(T(\phi) \phi \phi+\phi T(\phi) \phi+\phi \phi T(\phi)) \\
& =-(6 \lambda \Delta(\phi))^{2} \phi .
\end{aligned}
$$

From result (A.6), by assuming $6 \lambda \Delta(\phi) \neq 0$ (see discussion in subsection 2.5, in particular point (III)), one can check that the following two statements hold true:

1. The Freudenthal duality $\mathcal{F}(2.25)$ is an anti-involution in the FTS $\mathfrak{K}$, namely it squares to negative identity (cfr. (2.27) and point (I) of subsection 2.5):

$$
\mathcal{F}^{2} \equiv \mathcal{F} \circ \mathcal{F}=-I d
$$

2. The quartic polynomial $\Delta(\phi)$ (2.21) is invariant under the Freudenthal duality $\mathcal{F}(2.25)$, namely (cfr. $(2.26))$

$$
\Delta(\phi)=\Delta(\tilde{\phi}), \text { q.e.d. }
$$

\section{B Space-time symmetry of scalar kinetic term}

In order to prove the symmetry (3.31) of the FGT kinetic scalar term under the exchange of its space-time indices, one needs to re-write it only in terms of the $\mathfrak{K}$-valued scalar field $\phi(x)$, by recalling the definitions (2.21) and (2.25) of the quartic polynomial $\Delta(\phi)$ and of $F$-dual field $\widetilde{\phi}(x)$.

One starts by computing the FTS gauge covariant derivative of $\widetilde{\phi}(x)$, as follows:

$$
\begin{aligned}
D_{\mu} \tilde{\phi}(x) & =\operatorname{sgn}(\Delta(\phi)) \frac{1}{\sqrt{6}} D_{\mu}\left(\frac{T(\phi)}{\sqrt{|\lambda \Delta(\phi)|}}\right) \\
& =\frac{\operatorname{sgn}(\Delta(\phi))}{\sqrt{6|\lambda \Delta(\phi)|}}\left[3 \mathcal{L}_{\phi \phi} D_{\mu} \phi+6 \lambda\left\langle D_{\mu} \phi, \phi\right\rangle \phi+\frac{\left\langle D_{\mu} \phi, T(\phi)\right\rangle}{\Delta(\phi)} T(\phi)\right]
\end{aligned}
$$


As an aside, notice that the $\Delta(\phi)$ in the denominator of the last term does not have absolute signs attached to it. Plugging this expression into the kinetic term (prior to contraction with $\eta^{\mu \nu}$ ) yields its following explicit re-writing only in terms of $\phi(x)$ :

$$
\begin{aligned}
& \frac{1}{2}\left\langle D_{\mu} \phi, D_{\nu} \tilde{\phi}\right\rangle=\frac{\operatorname{sgn}(\Delta(\phi))}{2 \sqrt{6|\lambda \Delta(\phi)|}}[ 3\left\langle D_{\mu} \phi, \mathcal{L}_{\phi \phi} D_{\nu} \phi\right\rangle+6 \lambda\left\langle D_{\mu} \phi, \phi\right\rangle\left\langle D_{\nu} \phi, \phi\right\rangle \\
&\left.+\frac{1}{\Delta(\phi)}\left\langle D_{\mu} \phi, T(\phi)\right\rangle\left\langle D_{\nu} \phi, T(\phi)\right\rangle\right] .
\end{aligned}
$$

On the other hand, the second and third term of (B.2) are manifestly symmetric under $\mu \leftrightarrow \nu$, the symmetry of the first term can be proved as follows:

$$
\left\langle D_{\mu} \phi, \mathcal{L}_{\phi \phi} D_{\nu} \phi\right\rangle=-\left\langle\mathcal{L}_{\phi \phi} D_{\mu} \phi, D_{\nu} \phi\right\rangle=\left\langle D_{\nu} \phi, \mathcal{L}_{\phi \phi} D_{\mu} \phi\right\rangle,
$$

thus implying the result (3.31).

\section{Axioms of $\mathfrak{V}$}

As discussed in subsection 4.2, we report here the five axioms induced on $\mathfrak{V}$ by the five axioms $(o)$ - $(i v)$ of the algebra $\mathfrak{N}$ (in addition to the ones already introduced on $\mathfrak{V}$ for other physical reasons, such as the ones required by the Bose and/or Fermi statistics for the fields $\left.v_{I} \in \mathfrak{V}\right)$. In particular, in the proof of the no-go theorem in subsection 4.2, a crucial role is played by axioms $(B . i i i)$ and $(B . i i)$.

$(B . o)\left(v_{I}, v_{J}\right)_{\mathfrak{V}}=\left(v_{J}, v_{I}\right)_{\mathfrak{V}} ;$

(B.i) $\left[v_{I}, v_{J}, v_{K}\right]_{\mathfrak{V}}=\left[v_{J}, v_{I}, v_{K}\right]_{\mathfrak{V}} ;$

$(B . i i)\left(\phi_{I} \phi_{J} \phi_{K}\right) \otimes\left(\left[v_{I}, v_{J}, v_{K}\right]_{\mathfrak{V}}-\left[v_{I}, v_{K}, v_{J}\right]_{\mathfrak{V}}\right)$

$$
\begin{aligned}
= & \left\langle\phi_{J}, \phi_{K}\right\rangle \phi_{I} \otimes\left(2 \mu\left(v_{J}, v_{K}\right)_{\mathfrak{V}} \times v_{I}-2 \lambda\left[v_{I}, v_{J}, v_{K}\right]_{\mathfrak{V}}\right) \\
& +\left\langle\phi_{I}, \phi_{K}\right\rangle \phi_{J} \otimes\left(\mu\left(v_{I}, v_{K}\right)_{\mathfrak{V}} \times v_{J}-\lambda\left[v_{I}, v_{J}, v_{K}\right]_{\mathfrak{V}}\right) \\
& -\left\langle\phi_{I}, \phi_{J}\right\rangle \phi_{K} \otimes\left(\mu\left(v_{I}, v_{J}\right)_{\mathfrak{V}} \times v_{K}-\lambda\left[v_{I}, v_{J}, v_{K}\right]_{\mathfrak{V}}\right) ;
\end{aligned}
$$

(B. iii) $0=\left(\phi_{L} \phi_{M} \phi_{I}\right) \phi_{J} \phi_{K} \otimes\left(\left[v_{L}, v_{M},\left[v_{I}, v_{J}, v_{K}\right]_{\mathfrak{V}}\right]_{\mathfrak{V}}-\left[\left[v_{L}, v_{M}, v_{I}\right]_{\mathfrak{V}}, v_{J}, v_{K}\right]_{\mathfrak{V}}\right)$ $+\phi_{I}\left(\phi_{L} \phi_{M} \phi_{J}\right) \phi_{K} \otimes\left(\left[v_{L}, v_{M},\left[v_{I}, v_{J}, v_{K}\right]_{\mathfrak{V}}\right]_{\mathfrak{V}}-\left[v_{I},\left[v_{L}, v_{M}, v_{J}\right]_{\mathfrak{V}}, v_{K}\right]_{\mathfrak{V}}\right)$ $+\phi_{I} \phi_{J}\left(\phi_{L} \phi_{M} \phi_{K}\right) \otimes\left(\left[v_{L}, v_{M},\left[v_{I}, v_{J}, v_{K}\right]_{\mathfrak{V}}\right]_{\mathfrak{V}}-\left[v_{I}, v_{J},\left[v_{L}, v_{M}, v_{K}\right]_{\mathfrak{V}}\right]_{\mathfrak{V}}\right) ;$

$(B . i v)\left(\left[v_{L}, v_{M}, v_{I}\right]_{\mathfrak{V}}, v_{J}\right)_{\mathfrak{V}}+\left(v_{I},\left[v_{L}, v_{M}, v_{J}\right]_{\mathfrak{V}}\right)_{\mathfrak{V}}=0$.

Open Access. This article is distributed under the terms of the Creative Commons Attribution License which permits any use, distribution and reproduction in any medium, provided the original author(s) and source are credited. 


\section{References}

[1] Y. Nambu, Generalized Hamiltonian dynamics, Phys. Rev. D 7 (1973) 2405 [INSPIRE].

[2] R. Chatterjee and L. Takhtajan, Aspects of classical and quantum Nambu mechanics, Lett. Math. Phys. 37 (1996) 475 [hep-th/9507125] [InSPIRE].

[3] M. Günaydin, Quadratic Jordan formulation of quantum mechanics and construction of Lie (super)algebras from Jordan (super)algebras, Ann. Israel Phys. Soc. 3 (1980) 279, presented at $8^{\text {th }}$ Int. Colloq. on Group Theoretical Methods in Physics, Kiriat Anavim Israel March 25-29 1979 [INSPIRE].

[4] M. Günaydin, The exceptional superspace and the quadratic Jordan formulation of quantum mechanics, in Elementary particles and the universe: essays in honor of Murray Gell-Mann, Pasadena U.S.A., J. Schwarz ed., Cambridge University Press, Cambridge U.K. (1989), pg. 99.

[5] M. Günaydin, C. Piron and H. Ruegg, Moufang plane and octonionic quantum mechanics, Commun. Math. Phys. 61 (1978) 69 [INSPIRE].

[6] I. Bars and M. Günaydin, Construction of Lie algebras and Lie superalgebras from ternary algebras, J. Math. Phys. 20 (1979) 1977 [INSPIRE].

[7] I. Bars and M. Günaydin, Dynamical theory of subconstituents based on ternary algebras, Phys. Rev. D 22 (1980) 1403 [InSPIRE].

[8] M. Günaydin and S. Hyun, Ternary algebraic approach to extended superconformal algebras, Nucl. Phys. B 373 (1992) 688 [InSPIRE].

[9] M. Günaydin, Extended superconformal symmetry, Freudenthal triple systems and gauged WZW models, presented at the Gursey Memorial Conference I: on Strings and Symmetries, Istanbul Turkey June 6-10 1994 [hep-th/9502064] [INSPIRE].

[10] J. Bagger and N. Lambert, Gauge symmetry and supersymmetry of multiple M2-branes, Phys. Rev. D 77 (2008) 065008 [arXiv:0711.0955] [INSPIRE].

[11] A. Gustavsson, Algebraic structures on parallel M2-branes, Nucl. Phys. B 811 (2009) 66 [arXiv:0709.1260] [INSPIRE].

[12] J. Bagger, N. Lambert, S. Mukhi and C. Papageorgakis, Multiple membranes in M-theory,

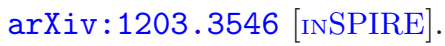

[13] P.-M. Ho, Y. Imamura, Y. Matsuo and S. Shiba, M5-brane in three-form flux and multiple M2-branes, JHEP 08 (2008) 014 [arXiv:0805.2898] [INSPIRE].

[14] M. Günaydin, G. Sierra and P. Townsend, Exceptional supergravity theories and the MAGIC square, Phys. Lett. B 133 (1983) 72 [INSPIRE].

[15] M. Günaydin, G. Sierra and P. Townsend, The geometry of $N=2$ Maxwell-Einstein supergravity and Jordan algebras, Nucl. Phys. B 242 (1984) 244 [InSPIRE].

[16] M. Günaydin, K. Koepsell and H. Nicolai, Conformal and quasiconformal realizations of exceptional Lie groups, Commun. Math. Phys. 221 (2001) 57 [hep-th/0008063] [InSPIRE].

[17] M. Günaydin and O. Pavlyk, Spectrum generating conformal and quasiconformal U-duality groups, supergravity and spherical vectors, JHEP 04 (2010) 070 [arXiv:0901.1646] [INSPIRE]. 
[18] M. Günaydin and O. Pavlyk, Quasiconformal realizations of $E_{6(6)}, E_{7(7)}, E_{8(8)}$ and $\mathrm{SO}(n+3, m+3), \mathcal{N} \geqslant 4$ supergravity and spherical vectors, Adv. Theor. Math. Phys. 13 (2009) [arXiv:0904.0784] [INSPIRE].

[19] M. Günaydin, Lectures on spectrum generating symmetries and U-duality in supergravity, extremal black holes, quantum attractors and harmonic superspace, Springer Proc. Phys. 134 (2010) [arXiv:0908.0374] [INSPIRE].

[20] L. Borsten, M. Duff, S. Ferrara, A. Marrani and W. Rubens, Explicit orbit classification of reducible Jordan algebras and Freudenthal triple systems, arXiv:1108.0908 [INSPIRE].

[21] L. Borsten, M. Duff, S. Ferrara, A. Marrani and W. Rubens, Small orbits, Phys. Rev. D 85 (2012) 086002 [arXiv:1108.0424] [INSPIRE].

[22] R.B. Brown, Groups of type $E_{7}, J$. Reine Angew. Math. 236 (1969) 79.

[23] L. Borsten, D. Dahanayake, M. Duff and W. Rubens, Black holes admitting a Freudenthal dual, Phys. Rev. D 80 (2009) 026003 [arXiv:0903.5517] [INSPIRE].

[24] S. Ferrara, A. Marrani and A. Yeranyan, Freudenthal duality and generalized special geometry, Phys. Lett. B 701 (2011) 640 [arXiv:1102.4857] [INSPIRE].

[25] A. Ceresole, S. Ferrara, A. Marrani and A. Yeranyan, Small black hole constituents and horizontal symmetry, JHEP 06 (2011) 078 [arXiv: 1104.4652] [INSPIRE].

[26] S. Ferrara and A. Marrani, Black holes and groups of type $E_{7}$, Pramana 78 (2012) 893 [arXiv:1112.2664] [INSPIRE].

[27] S. Ferrara and R. Kallosh, Creation of matter in the universe and groups of type $E_{7}$, JHEP 12 (2011) 096 [arXiv:1110.4048] [INSPIRE].

[28] S. Ferrara, R. Kallosh and A. Marrani, Degeneration of groups of type $E_{7}$ and minimal coupling in supergravity, JHEP 06 (2012) 074 [arXiv: 1202.1290] [INSPIRE].

[29] E. Cremmer and B. Julia, The $N=8$ supergravity theory. 1. The Lagrangian, Phys. Lett. B 80 (1978) 48 [INSPIRE].

[30] E. Cremmer and B. Julia, The SO(8) supergravity, Nucl. Phys. B 159 (1979) 141 [inSPIRE].

[31] C. Hull and P. Townsend, Unity of superstring dualities, Nucl. Phys. B 438 (1995) 109 [hep-th/9410167] [INSPIRE].

[32] J.D. Bekenstein, Black holes and entropy, Phys. Rev. D 7 (1973) 2333 [INSPIRE].

[33] S. Hawking, Gravitational radiation from colliding black holes, Phys. Rev. Lett. 26 (1971) 1344 [INSPIRE].

[34] A. Marrani et al., More on Freudenthal gauge theory and Jordan algebras, to appear (2012).

[35] J.R. Faulkner, On the geometry of inner ideals, J. Algebra 26 (1973) 1.

[36] P. de Medeiros, J. Figueroa-O'Farrill, E. Mendez-Escobar and P. Ritter, On the Lie-algebraic origin of metric 3-algebras, Commun. Math. Phys. 290 (2009) 871 [arXiv:0809.1086] [INSPIRE].

[37] P. de Medeiros, J. Figueroa-O'Farrill and E. Mendez-Escobar, Superpotentials for superconformal Chern-Simons theories from representation theory,

J. Phys. A 42 (2009) 485204 [arXiv:0908.2125] [INSPIRE].

[38] H. Freudenthal, Beziehungen der $E_{7}$ und $E_{8}$ zur Oktavenebene. II (in German), Nederl. Akad. Wetensch. Proc. Ser. A 57 (1954) 363 [Indag. Math. 16 (1954) 363]. 
[39] H. Freudenthal, Oktaven, Ausnahmegruppen und Oktavengeometrie (in German), Geom. Dedicata 19 (1985) 7.

[40] K. McCrimmon, The Freudenthal-Springer-Tits construction of exceptional Jordan algebras, Trans. Amer. Math. Soc. 139 (1969) 495.

[41] J.R. Faulkner, A construction of Lie algebras from a class of ternary algebras, Trans. Amer. Math. Soc. 155 (1971) 397.

[42] K. McCrimmon, A taste of Jordan algebras, Springer-Verlag New York Inc., New York U.S.A. (2004).

[43] K. Yamaguti and H. Asano, On the Freudenthal's construction of exceptional Lie algebras, Proc. Japan Acad. 51 (1975) 253.

[44] M.K. Gaillard and B. Zumino, Duality rotations for interacting fields, Nucl. Phys. B 193 (1981) 221 [inSPIRE].

[45] E.B. Dynkin, The maximal subgroups of the classical groups, Amer. Math. Soc. Transl. 6 (1957) 245.

[46] M. Lorente and B. Gruber, Classification of semisimple subalgebras of simple Lie algebras, J. Math. Phys. 13 (1972) 1639 [InSPIRE].

[47] J.C. Ferrar, Strictly regular elements in Freudenthal triple systems, Trans. Amer. Math. Soc. 174 (1972) 313.

[48] S. Okubo, Triple products and Yang-Baxter equation. 1. Octonionic and quaternionic triple systems, J. Math. Phys. 34 (1993) 3273 [hep-th/9212051] [INSPIRE].

[49] S. Okubo, Triple products and Yang-Baxter equation. 2. Orthogonal and symplectic ternary systems, J. Math. Phys. 34 (1993) 3292 [hep-th/9212052] [INSPIRE].

[50] I.L. Kantor, Certain generalizations of Jordan algebras, Trudy Sem. Vektor. Tenzor. Anal. 16 (1972) 407.

[51] J. Palmkvist, A realization of the Lie algebra associated to a Kantor triple system, J. Math. Phys. 47 (2006) 023505 [math. RA/0504544] [INSPIRE].

[52] I. Kantor and I. Skopets, Some results on Freudenthal triple systems, Sel. Math. Sov. 2 (1982) 293.

[53] L. Andrianopoli, R. D'Auria, S. Ferrara and M. Trigiante, Black-hole attractors in $N=1$ supergravity, JHEP 07 (2007) 019 [hep-th/0703178] [INSPIRE].

[54] S. Bellucci, S. Ferrara, M. Günaydin and A. Marrani, SAM lectures on extremal black holes in $D=4$ extended supergravity, Springer Proc. Phys. 134 (2010) 1 [arXiv:0905.3739] [INSPIRE].

[55] L. Manivel, Configurations of lines and models of Lie algebras, J. Algebra 304 (2006) 457 [math. AG/0507118].

[56] B. de Wit and H. Nicolai, Extended supergravity with local $\mathrm{SO}(5)$ invariance, Nucl. Phys. B 188 (1981) 98 [inSPIRE].

[57] B. de Wit, A. Tollsten and H. Nicolai, Locally supersymmetric $D=3$ nonlinear $\sigma$-models, Nucl. Phys. B 392 (1993) 3 [hep-th/9208074] [INSPIRE].

[58] M. Duff, J.T. Liu and J. Rahmfeld, Four-dimensional string-string-string triality, Nucl. Phys. B 459 (1996) 125 [hep-th/9508094] [INSPIRE]. 
[59] K. Behrndt, R. Kallosh, J. Rahmfeld, M. Shmakova and W.K. Wong, STU black holes and string triality, Phys. Rev. D 54 (1996) 6293 [hep-th/9608059] [INSPIRE].

[60] L. Andrianopoli, R. D'Auria and S. Ferrara, U invariants, black hole entropy and fixed scalars, Phys. Lett. B 403 (1997) 12 [hep-th/9703156] [INSPIRE].

[61] S. Ferrara, A. Gnecchi and A. Marrani, $D=4$ attractors, effective horizon radius and fake supergravity, Phys. Rev. D 78 (2008) 065003 [arXiv:0806.3196] [InSPIRE].

[62] D. Roest and H. Samtleben, Twin supergravities, Class. Quant. Grav. 26 (2009) 155001 [arXiv: 0904.1344] [INSPIRE].

[63] M. Günaydin, S. McReynolds and M. Zagermann, The R-map and the coupling of $N=2$ tensor multiplets in 5 and 4 dimensions, JHEP 01 (2006) 168 [hep-th/0511025] [INSPIRE].

[64] M. Henneaux and C. Teitelboim, Dynamics of chiral (selfdual) P forms, Phys. Lett. B 206 (1988) 650 [INSPIRE].

[65] J.H. Schwarz and A. Sen, Duality symmetric actions, Nucl. Phys. B 411 (1994) 35 [hep-th/9304154] [INSPIRE].

[66] C. Hillmann, $E_{7(7)}$ invariant Lagrangian of $D=4 N=8$ supergravity, JHEP 04 (2010) 010 [arXiv:0911.5225] [INSPIRE].

[67] E. Cremmer, B. Julia, H. Lü and C. Pope, Dualization of dualities. 1, Nucl. Phys. B 523 (1998) 73 [hep-th/9710119] [INSPIRE].

[68] L. Borsten, M. Duff, S. Ferrara and A. Marrani, Freudenthal dual Lagrangians, arXiv:1212.3254 [INSPIRE].

[69] J. Figueroa-O'Farrill, Simplicity in the Faulkner construction, J. Phys. A 42 (2009) 445206 [arXiv: 0905.4900] [INSPIRE].

[70] D. Gaiotto and E. Witten, Janus configurations, Chern-Simons couplings, and the theta-angle in $N=4$ super Yang-Mills theory, JHEP 06 (2010) 097 [arXiv:0804.2907] [INSPIRE].

[71] S.-S. Kim and J. Palmkvist, $\mathcal{N}=5$ three-algebras and 5-graded Lie superalgebras, J. Math. Phys. 52 (2011) 083502 [arXiv:1010.1457] [INSPIRE].

[72] J.R. Faulkner and J.C. Ferrar, Simple anti-Jordan pairs, Comm. Algebra 8 (1980) 993.

[73] A. Marrani, E. Orazi and F. Riccioni, Exceptional reductions, J. Phys. A 44 (2011) 155207 [arXiv: 1012.5797] [INSPIRE].

[74] J.C. Baez, The octonions, Bull. Am. Math. Soc. 39 (2002) 145 [math.RA/0105155] [inSPIRE].

[75] M. Yamazaki, Octonions, $G_{2}$ and generalized Lie 3-algebras, Phys. Lett. B 670 (2008) 215 [arXiv:0809.1650] [INSPIRE].

[76] C. Castro, Advances in ternary and octonionic gauge field theories, Int. J. Mod. Phys. A 26 (2011) 2997 [inSPIRE].

[77] C. Castro, On octonionic gravity, exceptional Jordan strings and nonassociative ternary gauge field theories, Int. J. Geom. Meth. Mod. Phys. 9 (2012) 1250021 [InSPIRE].

[78] P. Aschieri, S. Ferrara and B. Zumino, Duality rotations in nonlinear electrodynamics and in extended supergravity, Riv. Nuovo Cim. 31 (2008) 625 [arXiv:0807.4039] [INSPIRE].

[79] D. Cassani, S. Ferrara, A. Marrani, J.F. Morales and H. Samtleben, A special road to AdS vacua, JHEP 02 (2010) 027 [arXiv: 0911.2708] [INSPIRE]. 
[80] P. Jordan, J. von Neumann and E.P. Wigner, On an algebraic generalization of the quantum mechanical formalism, Annals Math. 35 (1934) 29 [INSPIRE].

[81] S. Cecotti, S. Ferrara and L. Girardello, Geometry of type II superstrings and the moduli of superconformal field theories, Int. J. Mod. Phys. A 4 (1989) 2475 [inSPIRE].

[82] P. Breitenlohner, D. Maison and G.W. Gibbons, Four-dimensional black holes from Kaluza-Klein theories, Commun. Math. Phys. 120 (1988) 295 [INSPIRE].

[83] A. Gustavsson, Selfdual strings and loop space Nahm equations, JHEP 04 (2008) 083 [arXiv: 0802.3456] [INSPIRE].

[84] A.L. Besse, Einstein manifolds, Springer-Verlag, Germany (1987).

[85] D.V. Alekseevsky and B.N. Kimelfeld, Structure of homogeneous Riemannian spaces with zero Ricci curvature, Funct. Anal. Appl. 9 (1975) 97.

[86] M. Duff and S. Ferrara, Generalized mirror symmetry and trace anomalies, Class. Quant. Grav. 28 (2011) 065005 [arXiv: 1009.4439] [INSPIRE].

[87] P. Levay and G. Sarosi, Hitchin functionals are related to measures of entanglement, Phys. Rev. D 86 (2012) 105038 [arXiv:1206.5066] [inSPIRE].

[88] J.M. Landsberg and L. Manivel, The projective geometry of Freudenthal's magic square, J. Algebra 239 (2001) 477 [math. AG/9908039]. 